Document downloaded from:

http://hdl.handle.net/10251/147417

This paper must be cited as:

Huang, W.; Khilko, D.; Kolyada, S.; Peris Manguillot, A.; Zhang, G. (2018). Finite Intersection Property and Dynamical Compactness. Journal of Dynamics and Differential Equations. 30(3):1221-1245. https://doi.org/10.1007/s10884-017-9600-8

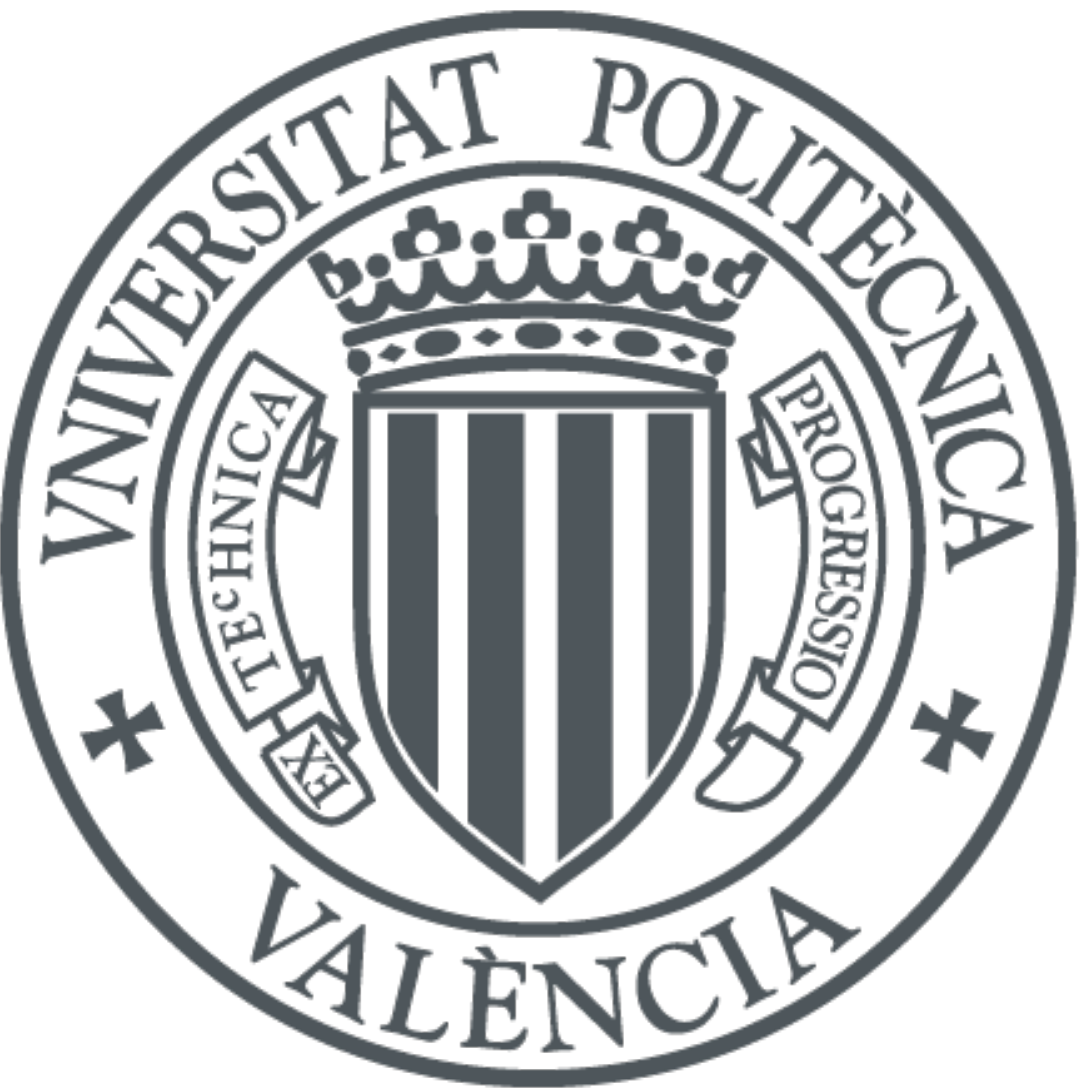

The final publication is available at

https://doi.org/10.1007/s10884-017-9600-8

Copyright Springer-Verlag

Additional Information 


\title{
FINITE INTERSECTION PROPERTY AND DYNAMICAL COMPACTNESS
}

\author{
WEN HUANG, DANYLO KHILKO, SERGIĬ KOLYADA, \\ ALFRED PERIS AND GUOHUA ZHANG
}

This paper is dedicated to Professor Ethan Akin on the occasion of his 70th birthday.

\begin{abstract}
Dynamical compactness with respect to a family as a new concept of chaoticity of a dynamical system was introduced and discussed in [23]. In this paper we continue to investigate this notion. In particular, we prove that all dynamical systems are dynamically compact with respect to a Furstenberg family if and only if this family has the finite intersection property. We investigate weak mixing and weak disjointness by using the concept of dynamical compactness. We also explore further difference between transitive compactness and weak mixing. As a byproduct, we show that the $\omega_{\mathcal{F}}$-limit and the $\omega$-limit sets of a point may have quite different topological structure. Moreover, the equivalence between multi-sensitivity, sensitive compactness and transitive sensitivity is established for a minimal system. Finally, these notions are also explored in the context of linear dynamics.
\end{abstract}

\section{INTRODUCTION}

By a (topological) dynamical system $(X, T)$ we mean a compact metric space $X$ with a metric $d$ and a continuous self-surjection $T$ of $X$. We say it is trivial if the space is a singleton. Throughout this paper, we are only interested in a nontrivial dynamical system, where the state space is a compact metric space without isolated points.

This paper is a continuation of the research carried out in [23], where the authors discussed a dynamical property called dynamical compactness and examined it firstly for transitive compactness. Some results of this paper can be considered as a contribution to an area of dynamical systems called dynamical topology, in which the topological properties of maps can be described in dynamical terms.

Let $\mathbb{Z}_{+}$be the set of all nonnegative integers and $\mathbb{N}$ the set of all positive integers. Before going on, let us recall the notion of a Furstenberg family from [1]. Denote by $\mathcal{P}=\mathcal{P}\left(\mathbb{Z}_{+}\right)$the set of all subsets of $\mathbb{Z}_{+}$. A subset $\mathcal{F} \subset \mathcal{P}$ is a (Furstenberg) family, if it is hereditary upward, that is, $F_{1} \subset F_{2}$ and $F_{1} \in \mathcal{F}$ imply $F_{2} \in \mathcal{F}$. Any subset $\mathcal{A}$ of $\mathcal{P}$ clearly generates a family $\{F \in \mathcal{P}: F \supset A$ for some $A \in \mathcal{A}\}$. Denote by $\mathcal{B}$ the family of all infinite subsets of $\mathbb{Z}_{+}$, and by $\mathcal{P}_{+}$the family of all nonempty subsets of $\mathbb{Z}_{+}$. For a family $\mathcal{F}$, the dual family of $\mathcal{F}$, denoted by $k \mathcal{F}$, is defined as

$$
\left\{F \in \mathcal{P}: F \cap F^{\prime} \neq \varnothing \text { for any } F^{\prime} \in \mathcal{F}\right\} \text {. }
$$

2010 Mathematics Subject Classification. Primary 37B05; Secondary 54H20, 47A16.

Key words and phrases. Dynamical topology; Dynamical compactness; Transitive compactness; Sensitive compactness; Topological weak mixing; Multi-sensitivity; Transitive sensitivity; Linear dynamics; Hypercyclic operators . 
A family $\mathcal{F}$ is proper if it is a proper subset of $\mathcal{P}$, that is, $\mathbb{Z}_{+} \in \mathcal{F}$ and $\varnothing \notin \mathcal{F}$. By a filter $\mathcal{F}$ we mean a proper family closed under intersection, that is, $F_{1}, F_{2} \in \mathcal{F}$ implies $F_{1} \cap F_{2} \in \mathcal{F}$. A filter is free if the intersection of all its elements is empty. We extend this concept, a family $\mathcal{F}$ is called free if the intersection of all elements of $\mathcal{F}$ is empty.

For any $F \in \mathcal{P}$, every point $x \in X$ and each subset $G \subset X$, we define $T^{F} x=$ $\left\{T^{i} x: i \in F\right\}, N_{T}(x, G)=\left\{n \in \mathbb{Z}_{+}: T^{n} x \in G\right\}$. The $\omega$-limit set of $x$ with respect to $\mathcal{F}$ (see [1]), or shortly the $\omega_{\mathcal{F}}$-limit set of $x$, denoted by $\omega_{\mathcal{F}}(x)^{1}$, is defined as

$$
\bigcap_{F \in \mathcal{F}} \overline{T^{F} x}=\left\{z \in X: N_{T}(x, G) \in k \mathcal{F} \text { for every neighborhood } G \text { of } z\right\} .
$$

Let us note that not always $\omega_{\mathcal{F}}(x)$ is a subset of the $\omega$-limit set $\omega_{T}(x)$, which is defined as

$$
\bigcap_{n=1}^{\infty} \overline{\left\{T^{k} x: k \geq n\right\}}=\left\{z \in X: N_{T}(x, G) \in \mathcal{B} \text { for every neighborhood } G \text { of } z\right\} .
$$

For instance, if each element of $\mathcal{F}$ contains 0 then any point $x \in \omega_{\mathcal{F}}(x)$. Nevertheless, if a family $\mathcal{F}$ is free, then $\omega_{\mathcal{F}}(x) \subset \omega_{T}(x)$ for any point $x \in X$ and if $(X, T)$ has a nonrecurrent point ${ }^{2}$, then the converse is true (see Proposition 2.2).

A dynamical system $(X, T)$ is called compact with respect to $\mathcal{F}$, or shortly $d y$ namically compact, if the $\omega_{\mathcal{F}}$-limit set $\omega_{\mathcal{F}}(x)$ is nonempty for all $x \in X$.

H. Furstenberg started a systematic study of transitive systems in his paper on disjointness in topological dynamics and ergodic theory [14], and the theory was further developed in [16] and [15]. Recall that the system $(X, T)$ is (topologically) transitive if $N_{T}\left(U_{1}, U_{2}\right)=\left\{n \in \mathbb{Z}_{+}: U_{1} \cap T^{-n} U_{2} \neq \varnothing\right\} \quad\left(=\left\{n \in \mathbb{Z}_{+}: T^{n} U_{1} \cap U_{2} \neq\right.\right.$ $\varnothing\}) \in \mathcal{P}_{+}$for any opene ${ }^{3}$ subsets $U_{1}, U_{2} \subset X$, equivalently, $N_{T}\left(U_{1}, U_{2}\right) \in \mathcal{B}$ for any opene subsets $U_{1}, U_{2} \subset X$.

In [23] the authors consider one of possible dynamical compactness - transitive compactness, and its relations with well-known chaotic properties of dynamical systems. Let $\mathcal{N}_{T}$ be the set of all subsets of $\mathbb{Z}_{+}$containing some $N_{T}(U, V)$, where $U, V$ are opene subsets of $X$. A dynamical system $(X, T)$ is called transitive compact, if for any point $x \in X$ the $\omega_{\mathcal{N}_{T}}$-limit set $\omega_{\mathcal{N}_{T}}(x)$ is nonempty, in other words, for any point $x \in X$ there exists a point $z \in X$ such that

$$
N_{T}(x, G) \cap N_{T}(U, V) \neq \varnothing
$$

for any neighborhood $G$ of $z$ and any opene subsets $U, V$ of $X$.

Let $(X, T)$ and $(Y, S)$ be two dynamical systems and $k \in \mathbb{N}$. The product system $(X \times Y, T \times S)$ is defined naturally, and denote by $\left(X^{k}, T^{(k)}\right)$ the product system of $k$ copies of the system $(X, T)$. Recall that the system $(X, T)$ is minimal if it does not admit a nonempty, closed, proper subset $K$ of $X$ with $T K \subset K$, and is weakly mixing if the product system $\left(X^{2}, T^{(2)}\right)$ is transitive. Any transitive compact system is obviously topologically transitive, and observe that each weakly mixing system is transitive compact ([4]). In fact, as it was shown in [23], each of notions are different in general and equivalent for minimal systems.

\footnotetext{
${ }^{1}$ Note that the notation $\omega_{\mathcal{F}}(x)$ used here is different from the one used in [1] (the notation $\omega_{\mathcal{F}}(x)$ used here is in fact $\omega_{k \mathcal{F}}(x)$ introduced in [1]). As this paper is a continuation of the research in [23], in order to avoid any confusion of notation or concept, we will follow the ones used in [23].

${ }^{2} \mathrm{~A}$ point $x \in X$ is called recurrent if $x \in \omega_{T}(x)$.

${ }^{3}$ Because we so often have to refer to open, nonempty subsets, we will call such subsets opene.
} 
Recall a very useful notion of weakly mixing subsets of a system, which was introduced in [9] and further discussed in [33] and [34]. The notion of weakly mixing subsets can be regraded as a local version of weak mixing. Among many very interesting properties let us mention just one of them - positive topological entropy of a dynamical system implies the existence of weakly mixing sets (see [29] for details). A nontrivial closed subset $A \subset X$ is called weakly mixing if for every $k \geq 2$ and any opene sets $U_{1}, \ldots, U_{k}, V_{1}, \ldots, V_{k}$ of $X$ with $U_{i} \cap A \neq \varnothing, V_{i} \cap A \neq \varnothing$, for any $i=1, \ldots, k$, one has that $\bigcap_{i=1}^{k} N_{T}\left(U_{i} \cap A, V_{i}\right) \neq \varnothing$. Let $A$ be a weakly mixing subset of $X$ and let $\mathcal{N}_{T}(A)$ be the set of all subsets of $\mathbb{Z}_{+}$containing some $N_{T}(U \cap A, V)$, where $U, V$ are opene subsets of $X$ intersecting $A$.

The notion of sensitivity was first used by Ruelle [37], which captures the idea that in a chaotic system a small change in the initial condition can cause a big change in the trajectory. According to the works by Guckenheimer [20], Auslander and Yorke [6] a dynamical system $(X, T)$ is called sensitive if there exists $\delta>0$ such that for every $x \in X$ and every neighborhood $U_{x}$ of $x$, there exist $y \in U_{x}$ and $n \in \mathbb{N}$ with $d\left(T^{n} x, T^{n} y\right)>\delta$. Such a $\delta$ is called a sensitive constant of $(X, T)$. Recently in [31] Moothathu initiated a way to measure the sensitivity of a dynamical system, by checking how large is the set of nonnegative integers for which the sensitivity occurs (see also [30]). For a positive $\delta$ and a subset $U \subset X$ define

$$
S_{T}(U, \delta)=\left\{n \in \mathbb{Z}_{+}: \text {there are } x_{1}, x_{2} \in U \text { such that } d\left(T^{n} x_{1}, T^{n} x_{2}\right)>\delta\right\} .
$$

A dynamical system $(X, T)$ is called multi-sensitive if there exists $\delta>0$ such that $\bigcap_{i=1}^{k} S_{T}\left(U_{i}, \delta\right) \neq \varnothing$ for any finite collection of opene $U_{1}, \ldots, U_{k} \subset X$. Such a $\delta$ is called a constant of multi-sensitivity of $(X, T)$.

Recall that a collection $A$ of subsets of a set $Y$ has the finite intersection property (FIP) if the intersection of all sets in any finite subcollection of $A$ is nonempty. The FIP is useful in formulating an alternative definition of compactness of a topological space: a topological space is compact if and only if every collection of closed subsets satisfying the FIP has a nonempty intersection itself (see, for instance $[13,26]$ ).

We recall that if $(X, T)$ is weakly mixing then the family $\mathcal{N}_{T}$ is a filter and hence has FIP. More generally, if $A$ a weakly mixing subset of $(X, T)$ then the family $\mathcal{N}_{T}(A)$ also has FIP. Denote by $\mathcal{S}_{T}(\delta)$ the set of all subsets of $\mathbb{Z}_{+}$containing $S_{T}(U, \delta)$ for some $\delta>0$ and opene $U \subset X$. If $(X, T)$ is a multi-sensitive system with a constant of multi-sensitivity $\delta>0$ then obviously the family $\mathcal{S}_{T}(\delta)$ has FIP. Since all of these families are also free, actually they have the strong finite intersection property (SFIP), i.e., if the intersection over any finite subcollection of the family is infinite (see Proposition 2.2).

In fact we can say more - the FIP is useful in characterizing the dynamical compactness (see Theorem 3.1).

Theorem FIP. All dynamical systems are dynamically compact with respect to $\mathcal{F}$ if and only if the family $\mathcal{F}$ has the finite intersection property.

We also introduce two new stronger versions of sensitivity: sensitive compactness and transitive sensitivity. We will call the system $(X, T)$ transitively sensitive if there exists $\delta>0$ such that $S_{T}(W, \delta) \cap N_{T}(U, V) \neq \varnothing$ for any opene subsets $U, V, W$ of $X$; and sensitive compact, if there exists $\delta>0$ such that for any point $x \in X$ the $\omega_{\mathcal{S}_{T}(\delta)}$-limit set $\omega_{\mathcal{S}_{T}(\delta)}(x)$ is nonempty, in other words, for any point $x \in X$ there 
exists a point $z \in X$ such that

$$
N_{T}(x, G) \cap S_{T}(U, \delta) \neq \varnothing
$$

for any neighborhood $G$ of $z$ and any opene $U$ of $X$.

The paper is organized as follows. In Section 2 we recall some basic concepts and properties used in later discussions from topological dynamics. In Section 3 we obtain some general results concerning dynamical compactness. In particular we show that all dynamical systems are dynamically compact with respect to a Furstenberg family if and only if this family has the finite intersection property (Theorem 3.1).

In Section 4 we discuss two stronger versions of sensitivity: transitive sensitivity and sensitive compactness. It was shown that each weakly mixing system is transitively sensitive (Proposition 4.5), and in fact we can characterize transitive sensitivity of a general dynamical system in terms of dynamical compactness (Proposition 4.3). Furthermore, all of the multi-sensitivity, sensitive compactness and transitive sensitivity are equivalent for a minimal system (Theorem 4.1). Even though each minimal transitive compact system is multi-sensitive, there are many minimal multi-sensitive systems which are not transitive compact. We recall that the sensitivity of a dynamical system can be lifted up from a factor to an extension by an almost open factor map between transitive systems by [17, Corollary 1.7]. We prove that the transitive sensitivity can be lifted up to an extension from a factor by an almost one-to-one factor map and that the transitive sensitivity is projected from an extension to the sensitivity of a factor by a weakly almost one-to-one factor map (Lemma 4.4).

In Section 5 we show that dynamical compactness can be used to characterize the weak disjointness of dynamical systems (Theorem 5.2). We also extend the result of Jian $\mathrm{Li}$ [28]: weak mixing implies $\mathcal{F}_{i p}$-point transitivity in terms of transitive compactness (Proposition 5.4).

In Section 6 the further difference between weak mixing and transitive compactness is explored. Precisely, there is a totally transitive, non weakly mixing, transitive compact system (Theorem 6.1); and in fact any compact metric space can be realized as the $\omega_{\mathcal{N}_{T}}$-limit set of a non totally transitive, transitive compact system $(X, T)$ (Theorem 6.4). As a byproduct, we show that the $\omega_{\mathcal{N}_{T}}$-limit sets and the $\omega$-limit sets have quite different topological structures for a general dynamical compact system $(X, T)$. At the end of this section we add one more chaotical property of transitive compact systems (in additional to already known from [23]): transitive compactness implies Li-Yorke chaos (Proposition 6.6).

In Section 7 we consider the dynamics of linear operators on infinite dimensional spaces in relation to the properties studied in previous sections. In particular, we show the equivalence of the topological weak mixing property with a weak version of transitive compactness (Theorem 7.1). Some results on sensitivity are also obtained.

Acknowledgements. The first and third authors acknowledge the hospitality of the School of Mathematical Sciences of the Fudan University, Shanghai. The third author also acknowledges the hospitality of the of the Max-Planck-Institute für Mathematik (MPIM) in Bonn, Departament de Matemàtica Aplicada of the Universitat Politècnica de València and the Department of Mathematics of the Chinese University of Hong Kong. 
We thank the referees for careful reading and constructive comments that have resulted in substantial improvements to this paper.

The first author was supported by NNSF of China (11225105, 11431012); the fourth author was supported by MINECO, Projects MTM2013-47093-P and MTM2016-75963-P, and by GVA, Project PROMETEOII/2013/013; and the fifth author was supported by NNSF of China (11671094).

\section{Preliminaries}

In this section we recall standard concepts and results used in later discussions.

2.1. Basic concepts in topological dynamics. Recall that $x \in X$ is a fixed point if $T x=x$, and an $\mathcal{F}$-transitive point of $(X, T)[28]$ if $N_{T}(x, U) \in \mathcal{F}$ for any opene subset $U$ of $X$. It is a trivial observation that if a family $\mathcal{F}$ admits an $\mathcal{F}$ transitive dynamical system $(X, T)$ without isolated points, then $\mathcal{F}$ is free. Since $k(k \mathcal{F})=\mathcal{F}$, it is easy to see that $x \in X$ is an $\mathcal{F}$-transitive point of $(X, T)$ if and only if $\omega_{k \mathcal{F}}(x)=X$. Denote by $\operatorname{Tran}_{\mathcal{F}}(X, T)$ the set of all $\mathcal{F}$-transitive points of $(X, T)$. The system $(X, T)$ is $\mathcal{F}$-point transitive if $\operatorname{Tran}_{\mathcal{F}}(X, T) \neq \varnothing$, and is $\mathcal{F}$-transitive if $N_{T}(U, V) \in \mathcal{F}$ for any opene subsets $U, V$ of $X$. Write $\operatorname{Tran}(X, T)=\operatorname{Tran}_{\mathcal{P}_{+}}(X, T)$ for short, and we also call the point $x$ transitive if $x \in \operatorname{Tran}(X, T)$, equivalently, its

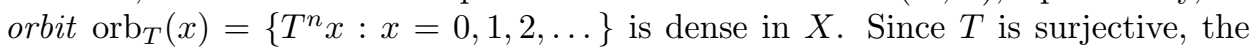
system $(X, T)$ is transitive if and only if $\operatorname{Tran}(X, T)$ is a dense $G_{\delta}$ subset of $X$.

In general, a subset $A$ of $X$ is $T$-invariant if $T A=A$, and positively $T$-invariant if $T A \subset A$. If $A$ is a closed, nonempty, $T$-invariant subset then $\left(A,\left.T\right|_{A}\right)$ is called the associated subsystem. A minimal subset of $X$ is a closed, nonempty, $T$-invariant subset such that the associated subsystem is minimal. Clearly, $(X, T)$ is minimal if and only if $\operatorname{Tran}(X, T)=X$, if and only if it admits no a proper, closed, nonempty, positively $T$-invariant subset. A point $x \in X$ is called minimal if it lies in some minimal subset. In this case, in order to emphasize the underlying system $(X, T)$ we also say that $x \in X$ is a minimal point of $(X, T)$. Zorn's Lemma implies that every closed, nonempty, positively $T$-invariant set contains a minimal set.

A pair of points $x, y \in X$ is called proximal if $\liminf _{n \rightarrow \infty} d\left(T^{n} x, T^{n} y\right)=0$. In this case each of points from the pair is said to be proximal to another. Denote by $\operatorname{Prox}_{T}(X)$ the set of all proximal pairs of points. For each $x \in X$, denote by $\operatorname{Prox}_{T}(x)$, called the proximal cell of $x$, the set of all points which are proximal to $x$. Recall that a dynamical system $(X, T)$ is called proximal if $\operatorname{Prox}_{T}(X)=X \times X$. The system $(X, T)$ is proximal if and only if $(X, T)$ has the unique fixed point, which is the only minimal point of $(X, T)$ (e.g. see [4]).

The opposition to the notion of sensitivity is the concept of equicontinuity. Recall that $x \in X$ is an equicontinuity point of $(X, T)$ if for every $\varepsilon>0$ there exists a $\delta>0$ such that $d\left(x, x^{\prime}\right)<\delta$ implies $d\left(T^{n} x, T^{n} x^{\prime}\right)<\varepsilon$ for any $n \in \mathbb{Z}_{+}$. Denote by $\operatorname{Eq}(X, T)$ the set of all equicontinuity points of $(X, T)$. The system $(X, T)$ is called equicontinuous if $\mathrm{Eq}(X, T)=X$. Each dynamical system admits a maximal equicontinuous factor. Recall that by a factor map $\pi:(X, T) \rightarrow(Y, S)$ between dynamical systems $(X, T)$ and $(Y, S)$, we mean that $\pi: X \rightarrow Y$ is a continuous surjection with $\pi \circ T=S \circ \pi$. In this case, we call $\pi:(X, T) \rightarrow(Y, S)$ an extension; and $(X, T)$ an extension of $(Y, S),(Y, S)$ a factor of $(X, T)$.

2.2. Basic concepts of Furstenberg families. In this subsection we recall from [1] basic concepts about Furstenberg families. 
Let $F \in \mathcal{P}$. Recall that a subset $F$ is thick if it contains arbitrarily long runs of positive integers. Denote by $\mathcal{F}_{\text {thick }}$ the set of all thick subsets of $\mathbb{Z}_{+}$, and define $\mathcal{F}_{\text {syn }}=k \mathcal{F}_{\text {thick }}$. Each element of $\mathcal{F}_{\text {syn }}$ is said to be syndetic, equivalently, $F$ is syndetic if and only if there is $N \in \mathbb{N}$ such that $\{i, i+1, \ldots, i+N\} \cap F \neq \varnothing$ for every $i \in \mathbb{Z}_{+}$. We say that $F$ is thickly syndetic if for every $N \in \mathbb{N}$ the positions where length $N$ runs begin form a syndetic set. Denote by $\mathcal{F}_{\text {cof }}$ the set of all cofinite subsets of $\mathbb{Z}_{+}$. Note that by the classic result of Gottschalk a point $x \in X$ is minimal if and only if $N_{T}(x, U)=\left\{n \in \mathbb{Z}_{+}: T^{n} x \in U\right\}$ is syndetic for any neighborhood $U$ of $x$. Hence, for any minimal system $(X, T)$, the subset $N_{T}(U, V)$ is syndetic for any opene subsets $U, V$ of $X$.

Recall that a family $\mathcal{F}$ is proper if it is a proper subset of $\mathcal{P}$, that is, $\mathbb{Z}_{+} \in \mathcal{F}$ and $\varnothing \notin \mathcal{F}$. By a filter $\mathcal{F}$ we mean a proper family closed under intersection, that is, $F_{1}, F_{2} \in \mathcal{F}$ implies $F_{1} \cap F_{2} \in \mathcal{F}$. For families $\mathcal{F}_{1}$ and $\mathcal{F}_{2}$, we define the family $\mathcal{F}_{1} \cdot \mathcal{F}_{2}:=\left\{F_{1} \cap F_{2}: F_{1} \in \mathcal{F}_{1}, F_{2} \in \mathcal{F}_{2}\right\}$ and call it the interaction of $\mathcal{F}_{1}$ and $\mathcal{F}_{2}$. Thus we have $\mathcal{F}_{1} \cup \mathcal{F}_{2} \subset \mathcal{F}_{1} \cdot \mathcal{F}_{2}$; and it is easy to check that $\mathcal{F}$ is a filter if and only if $\mathcal{F}=\mathcal{F} \cdot \mathcal{F}$, and $\mathcal{F}_{1} \cdot \mathcal{F}_{2}$ is proper if and only if $\mathcal{F}_{2} \subset k \mathcal{F}_{1}$.

For each $i \in \mathbb{Z}_{+}$, we define $g^{i}: \mathbb{Z}_{+} \rightarrow \mathbb{Z}_{+}, j \mapsto i+j$. Let $\mathcal{F}$ be a family. Recall that $\mathcal{F}$ is positively invariant if for every $i \in \mathbb{Z}_{+}, F \in \mathcal{F}$ implies $g^{i}(F) \in$ $\mathcal{F}$; negatively invariant if for every $i \in \mathbb{Z}_{+}, F \in \mathcal{F}$ implies $g^{-i}(F) \in \mathcal{F}$, where $g^{-i}(F)=\left(g^{i}\right)^{-1}(F)=\{j-i: j \in F, j \geq i\}$; and translation invariant if it is both positively and negatively invariant, equivalently, for every $i \in \mathbb{Z}_{+}, F \in \mathcal{F}$ if and only if $g^{-i}(F) \in \mathcal{F}$.

As $g^{-i}\left(g^{i} A\right)=A$ and $g^{i}\left(g^{-i} A\right) \subset A$ for any $i \in \mathbb{Z}_{+}$, it is easy to obtain that the family $\mathcal{F}$ is positively (negatively, translation, respectively) invariant if and only if $k \mathcal{F}$ is negatively (positively, translation, respectively) invariant (see for example $[1$, Proposition 2.5.b]). And then we have:

Proposition 2.1. Let $x \in X$. Then $T \omega_{\mathcal{F}}(x) \subset \omega_{\mathcal{F}}(T x)$. Additionally, if $\mathcal{F}$ is negatively (positively, translation, respectively) invariant then $\omega_{\mathcal{F}}(T x) \subset(\supset,=$, respectively) $\omega_{\mathcal{F}}(x)$.

Proof. Since the other items are alternative versions of [1, Proposition 3.6] in our notations, it suffices to prove that if $\mathcal{F}$ is positively invariant then $\omega_{\mathcal{F}}(T x) \supset \omega_{\mathcal{F}}(x)$.

For each $y \in \omega_{\mathcal{F}}(x)$ take an arbitrary neighborhood $U$ of $y$, and let $F \in \mathcal{F}$. Then $g^{1}(F)=\left\{i+1 \in \mathbb{Z}_{+}: i \in F\right\} \in \mathcal{F}$ as $\mathcal{F}$ is positively invariant, and hence $N_{T}(x, U) \cap g^{1}(F) \neq \varnothing$, thus $\varnothing \neq g^{-1}\left(N_{T}(x, U) \cap g^{1}(F)\right)=N_{T}(T x, U) \cap F$. It follows $y \in \omega_{\mathcal{F}}(T x)$ from the arbitrariness of $U$ and $F$, which finishes the proof.

Proposition 2.2. Let $(X, T)$ be a dynamical system and let $\mathcal{F}$ be a family.

(i) If $\mathcal{F}$ is free, then $\omega_{\mathcal{F}}(x) \subset \omega_{T}(x)$ for any $x \in X$. Moreover, if $(X, T)$ has a nonrecurrent point, then the converse implication is true.

(ii) If $\mathcal{F}$ is free and has FIP then it has SFIP.

Proof. (i) Suppose $\omega_{\mathcal{F}}(x) \neq \varnothing$ and take a point $y \in \omega_{\mathcal{F}}(x):=\bigcap_{F \in \mathcal{F}} \overline{T^{F} x}$. Let us show that $y \in \omega_{T}(x)$. If $\operatorname{orb}_{T}(x)$ is a finite set (equivalently $T^{i} x=T^{j} x$ for some $0 \leq i<j$ ) then for each $F \in \mathcal{F}$ there exists $n_{F} \in F$ with $y=T^{n_{F}} x$. Since $\mathcal{F}$ is free, there exist different elements $F$ and $F^{\prime}$ of $\mathcal{F}$ such that $n_{F}<n_{F^{\prime}}$, which implies $y=T^{n_{F}+k\left(n_{F^{\prime}}-n_{F}\right)} x$ for all $k \in \mathbb{Z}_{+}$and hence $y \in \omega_{T}(x)$.

Now assume that $T^{i} x \neq T^{j} x$ whenever $0 \leq i \neq j$. Since $\mathcal{F}$ is free, $\bigcap_{F \in \mathcal{F}} T^{F} x=$ $\varnothing$. Otherwise there is $m \in \mathbb{Z}_{+}$such that $T^{m} x \in T^{F} x$ for all $F \in \mathcal{F}$, in other words 
$m \in \bigcap_{F \in \mathcal{F}} F$, a contradiction. It means that there is $F \in \mathcal{F}$ such that $y \notin T^{F} x$. So $y \in \overline{T^{F} x} \backslash T^{F} x$, and $F$ is infinite. Therefore there exists an infinite sequence $T^{n_{1}} x, \ldots, T^{n_{i}} x, \ldots$, which converges to $y$. Hence $y \in \omega_{T}(x)$.

Now, let $(X, T)$ be a dynamical system with a nonrecurrent point $x_{0} \in X$, let $\mathcal{F}$ be a family and $\omega_{\mathcal{F}}(x) \subset \omega_{T}(x)$ for any $x \in X$. Suppose $\mathcal{F}$ is not free. It means there is a $k \in \mathbb{Z}_{+}$that lies in each element of $\mathcal{F}$. Then obviously that $T^{k}(x) \in \omega_{\mathcal{F}}(x)$ for any $x \in X$. Since $x_{0}$ is nonrecurrent, $x_{0} \notin \omega_{T}\left(x_{0}\right) \neq X$. It is well known that $\omega_{T}(x)$ is $T$-invariant, therefore $\omega_{T}(y)=\omega_{T}\left(x_{0}\right)$ for any $y \in\left\{T^{-i}\left(x_{0}\right): i \in \mathbb{Z}_{+}\right\}$, and $y \notin \omega_{T}(y)$. Take a point $x_{k} \in X$ with $T^{k}\left(x_{k}\right)=x_{0}$. As we know $x_{0} \in \omega_{\mathcal{F}}\left(x_{k}\right)$. But $\omega_{\mathcal{F}}\left(x_{k}\right) \subset \omega_{T}\left(x_{k}\right)=\omega_{T}\left(x_{0}\right)$, a contradiction.

(ii) Suppose that for some $F_{1}, \ldots F_{k} \in \mathcal{F} \bigcap_{i=1}^{k} F_{i}=\left\{n_{1}, n_{2}, \ldots n_{m}\right\}$. Since $\mathcal{F}$ is free for each $k \in \mathbb{Z}_{+}$there is $G_{k} \in \mathcal{F}$ such that $k \notin G_{k}$. Then $\bigcap_{i=1}^{k} F_{i} \cap \bigcap_{j=1}^{m} G_{n_{j}}=$ $\varnothing$, contradiction.

2.3. The concept of an almost one-to-one map. Let $\phi: X \rightarrow Y$ be a continuous surjective map from a compact metric space $X$ onto a compact Hausdorff space $Y$. Recall that $\phi$ is almost open if $\phi(U)$ has a nonempty interior in $Y$ for any opene $U \subset X$. Note that each factor map between minimal systems is almost open [5, Theorem 1.15], in particular, for a minimal system $(X, T)$ the map $T: X \rightarrow X$ is almost open [27]. Denote by $Y_{0} \subset Y$ the set of all points $y \in Y$ whose fiber is a singleton. Then $Y_{0}$ is a $G_{\delta}$ subset of $Y$, because

$$
Y_{0}=\left\{y \in Y: \phi^{-1}(y) \text { is a singleton }\right\}=\bigcap_{n \in \mathbb{N}}\left\{y \in Y: \operatorname{diam}\left(\phi^{-1}(y)\right)<\frac{1}{n}\right\}
$$

and the map $y \mapsto \operatorname{diam}\left(\phi^{-1}(y)\right)$ is upper semi-continuous. Here, we denote by $\operatorname{diam}(A)$ the diameter of a subset $A \subset X$. Recall that the function $f: Y \rightarrow \mathbb{R}_{+}$is upper semi-continuous if $\limsup _{y \rightarrow y_{0}} f(y) \leq f\left(y_{0}\right)$ for each $y_{0} \in Y$. Denote by $X_{0} \subset X$ the set of all points $x \in X$ such that the pre-image of $\phi(x)$ is a singleton. Then $X_{0}=\pi^{-1}\left(Y_{0}\right)$ is a $G_{\delta}$ subset of $X$.

We call $\phi$ weakly almost one-to-one if $Y_{0}$ is dense in $Y$, and almost one-to-one ${ }^{4}$ if $X_{0}$ is dense in $X$. It is not hard to show that: if $\phi$ is weakly almost one-to-one, then for any $\delta>0$ and any opene subset $U$ of $Y$ there exists opene $V \subset U$ with $\operatorname{diam}\left(\phi^{-1} V\right)<\delta$; and if $\phi$ is almost one-to-one, then for any opene subset $U^{*}$ of $X$ there exists an opene subset $V^{*}$ of $Y$ with $\phi^{-1} V^{*} \subset U^{*}$. Clearly almost one-to-one is much stronger than weakly almost one-to-one. For example, let $X$ be the closed unit interval, define $T(x)=2 x$ for $x \in\left[0, \frac{1}{2}\right]$ and $T(x)=1$ for $x \in\left[\frac{1}{2}, 1\right]$, and then $T: X \rightarrow X$ is clearly not almost one-to-one but weakly almost one-to-one.

For each minimal system $(X, T)$, the map $T: X \rightarrow X$ is weakly almost oneto-one [27, Theorem 2.7], and in fact almost one-to-one [24, Proposition 2.3]. The following result characterizes the relationship between weakly almost one-to-one and almost one-to-one, which extends [24, Proposition 2.3].

Proposition 2.3. Let $\phi: X \rightarrow Y$ be a continuous surjective map from a compact metric space $X$ onto a compact Hausdorff space $Y$. Then $\phi$ is almost one-to-one if and only if it is almost open and weakly almost one-to-one.

\footnotetext{
${ }^{4}$ Here we use the concept of almost one-to-one following [3], and the concept of almost oneto-one used in $[12,24,27]$ is in fact our weakly almost one-to-one.
} 
Proof. Firstly assume that $\phi$ is almost one-to-one. Let $U \subset X$ be an arbitrary opene subset. Take $x_{0} \in U$ such that the pre-image of $\phi\left(x_{0}\right)$ is a singleton. From this it is easy to see that $\phi\left(x_{0}\right)$ is contained in the interior of $\phi(U)$. This implies that $\phi$ is almost open. The map $\phi$ is clearly weakly almost one-to-one.

Now assume that $\phi$ is almost open and weakly almost one-to-one. Let $U \subset X$ be an arbitrary opene subset. Since $\phi$ is almost open, $\phi(U)$ has a nonempty interior in $Y$, and then $\phi^{-1}\left(y_{0}\right)$ is a singleton for some $y_{0} \in \phi(U)$, as $\phi$ is weakly almost one-to-one. This shows $U \cap X_{0} \neq \varnothing$, which finishes the proof.

As a direct corollary, we have:

Corollary 2.4. Let $\phi: X \rightarrow Y$ and $\pi: Y \rightarrow Z$ be continuous surjective maps between compact metric spaces. Then the composition map $\pi \circ \phi: X \rightarrow Z$ is almost one-to-one if and only if both $\phi$ and $\pi$ are almost one-to-one.

Proof. Denote by $X_{0}\left(X_{1}\right.$, respectively) the set of all points $x \in X$ such that the pre-image of $(\pi \circ \phi)(x)\left(\phi(x)\right.$, respectively) is a singleton. Denote by $Z_{0}\left(Z_{1}\right.$, respectively) the set of all points $z \in Z$ whose $\pi \circ \phi$-fibers ( $\pi$-fibers, respectively) are singletons. All of them are $G_{\delta}$ subsets. Moreover, $X_{0}=X_{1} \cap \phi^{-1}\left(\pi^{-1} Z_{1}\right)$. In fact, $x \in X_{0}$ if and only if $\{x\}=(\pi \circ \phi)^{-1}(\pi \circ \phi(x))=\phi^{-1}\left(\pi^{-1}(\pi(\phi x))\right)$, if and only if $\pi^{-1}(\pi(\phi x))=\{\phi(x)\}$ and $\phi^{-1}(\phi x)=\{x\}$, if and only if $\pi(\phi x) \in Z_{1}$ and $x \in X_{1}$.

First assume that $\pi \circ \phi$ is almost one-to-one, and then by Proposition 2.3: $X_{0}$ is a dense subset of $X, Z_{0}$ is a dense subset of $Z$ and the map $\pi \circ \phi$ is almost open. Note that $X_{0} \subset X_{1}$ and $Z_{0} \subset Z_{1}$, we have that $X_{1}$ is dense in $X$ and $Z_{1}$ is dense in $Z$. Hence $\phi$ is almost one-to-one. Furthermore, as the map $\pi \circ \phi$ is almost open, for any opene $V \subset Y$ one has that $\pi(V)=(\pi \circ \phi)\left(\phi^{-1} V\right)$ has a nonempty interior in $Z$, which implies that $\pi$ is almost one-to-one by Proposition 2.3 .

Now assume that both $\phi$ and $\pi$ are almost one-to-one. Then $X_{1}$ is a dense $G_{\delta}$ subset of $X$ and $Z_{1}$ is a dense $G_{\delta}$ subset of $Z$. Moreover, by Proposition 2.3 both $\phi$ and $\pi$ are almost open, and then the continuous surjection $\pi \circ \phi$ is also almost open, which implies that $(\pi \circ \phi)^{-1}\left(Z_{1}\right)$ is also a dense $G_{\delta}$ subset of $X$. Thus, $X_{0}=X_{1} \cap \phi^{-1}\left(\pi^{-1} Z_{1}\right)$ is a dense $G_{\delta}$ subset of $X$, that is, the composition map $\pi \circ \phi: X \rightarrow Z$ is almost one-to-one. This finishes the proof.

Let $\pi:(X, T) \rightarrow(Y, S)$ be a factor map between dynamical systems. If the map $\pi: X \rightarrow Y$ is almost one-to-one (weakly almost one-to-one, respectively), then we also call $(X, T)$ an almost one-to-one extension (a weakly almost oneto-one extension, respectively) of $(Y, S)$. The main result of [24] states that a minimal system is either multi-sensitive or a weakly almost one-to-one extension of its maximal equicontinuous factor. This is an analog of the well-known AuslanderYorke dichotomy theorem: a minimal system is either sensitive or equicontinuous.

2.4. Symbolic dynamics. Let $A$ be a nonempty finite set. We call $A$ the alphabet and elements of $A$ are symbols. The full (one-sided) $A$-shift is defined as

$$
\Sigma=\left\{x=\left\{x_{i}\right\}_{i=0}^{\infty}: x_{i} \in A \text { for all } i \in \mathbb{Z}_{+}\right\},
$$

where we equip $A$ with the discrete topology and $\Sigma$ with the product topology, and the shift map $\sigma: \Sigma \rightarrow \Sigma$ is a continuous surjection given by

$$
x=\left\{x_{i}\right\}_{i=0}^{\infty} \mapsto \sigma x=\left\{x_{i+1}\right\}_{i=0}^{\infty},
$$

that is, $\sigma(x)$ is the sequence obtained by dropping the first symbol of $x$. Usually we write an element of $\Sigma$ as $x=\left\{x_{i}\right\}_{i=0}^{\infty}=x_{0} x_{1} x_{2} x_{3} \ldots$ 
A block $w$ over $\Sigma$ is a finite sequence of symbols and its length is the number of its symbols (denoted by $|w|$ ). An $n$-block stands for a block of length $n$. In general we are only interested in a block $w$ with $|w| \geq 1$ if without any special statement, and denote by $\Sigma^{*}$ the set of all blocks over $\Sigma$. The block $w$ is a subblock of a block $v=v_{1} \ldots v_{m}$ with $v_{1}, \ldots, v_{m} \in A$ if there exists $1 \leq i \leq j \leq m$ with $w=v_{i} \ldots v_{j}$. The concatenation of two blocks $u=a_{1} \ldots a_{k}$ and $v=b_{1} \ldots b_{l}$ is the block $u v=a_{1} \ldots a_{k} b_{1} \ldots b_{l}$. We write $u^{n}$ for the concatenation of $n \geq 1$ copies of a block $u$ and $u^{\infty}$ for the sequence $u u u \cdots \in \Sigma$. By $x_{[i, j]}$ we denote the block $x_{i} x_{i+1} \ldots x_{j}$, where $0 \leq i \leq j$ and $x=\left\{x_{k}\right\}_{k=0}^{\infty} \in \Sigma$. The subset $X \subset \Sigma$ is called a subshift if it is a closed, nonempty, $\sigma$-invariant subset of $\Sigma$. A cylinder of an $n$-block $w \in \Sigma^{*}$ in a subshift $X$ is the set $C[w]=\left\{x \in X: x_{[0, n-1]}=w\right\}$. The collection of all cylinders forms a basis of the topology of $X$.

\section{DyNAMiCAL COMPACTNESS With RESPECT TO AN ARBITRARY FAMILY}

Recall that a family $\mathcal{F}$ has the finite intersection property (FIP) if the intersection of all sets in any finite subcollection of $\mathcal{F}$ is nonempty. The following theorem shows that the FIP is useful in characterizing the dynamical compactness.

Theorem 3.1. All dynamical systems are dynamically compact with respect to the family $\mathcal{F}$ if and only if $\mathcal{F}$ has the finite intersection property.

Proof. Sufficiency. Suppose that $\mathcal{F}$ has FIP. Take arbitrary dynamical system $(X, T)$ and let $x \in X$. Obviously the family $\left\{\overline{T^{F} x}: F \in \mathcal{F}\right\}$ also has FIP, and then by compactness of $X$ the family $\left\{\overline{T^{F} x}: F \in \mathcal{F}\right\}$ has a nonempty intersection itself, i.e., $\omega_{\mathcal{F}}(x) \neq \varnothing$. Thus $(X, T)$ is dynamically compact with respect to $\mathcal{F}$.

Necessity. Suppose that the family $\mathcal{F}$ has no FIP, and then there is a collection $\left\{F_{1}, \ldots, F_{k}\right\} \subset \mathcal{F}$ with $\bigcap_{i=1}^{k} F_{i}=\varnothing$. Let $A=\left\{a_{1}, \ldots, a_{k}\right\}$ be an alphabet and let $(X, T):=(\Sigma, \sigma)$ be the full (one-sided) $A$-shift. We are going to define a point $x \in X$ with $\omega_{\mathcal{F}}(x)=\varnothing$. Let $x_{0}=a_{1}$. For any $n \geq 1$ there is $i$ with $n \notin F_{i}$, else the intersection of $F_{1}, \ldots, F_{k}$ would be nonempty. Then define $x_{n}:=a_{i}$. Finally, let $x=x_{0} x_{1} x_{2} x_{3} \ldots$ and the construction is finished.

Assume the contrary that we can take $z \in \omega_{\mathcal{F}}(x)$, and that $z$ begins with $a_{i} \in A$. Take $G_{z}=C\left[a_{i}\right]$. As $z \in \omega_{\mathcal{F}}(x)$ we have $N_{T}\left(x, G_{z}\right) \cap F_{i} \neq \varnothing$. But if $n \in N_{T}\left(x, G_{z}\right)$, then $x_{n}=a_{i}$ and so $n \notin F_{i}$ by the construction, a contradiction.

As we have mentioned in Introduction, any filter has FIP; if $(X, T)$ is weakly mixing then the family $\mathcal{N}_{T}$ is a filter; if $A$ a weakly mixing subset of $(X, T)$ then the family $\mathcal{N}_{T}(A)$ has FIP; and if $(X, T)$ is a multi-sensitive system with a constant of multi-sensitivity $\delta>0$ then the family $\mathcal{S}_{T}(\delta)$ also has FIP.

Let $\mathcal{F}$ have the finite intersection property. Then there exists an ultrafilter $\mathcal{U}$ (in $\mathcal{P}$ ) such that $\mathcal{F} \subset \mathcal{U}$. This result is known as Ultrafilter Lemma (see details and proof in [21]). Recall that an ultrafilter is maximal among all proper filters. As a consequence of this fact we have a natural open question:

Question A. Let $(X, T)$ be a dynamically compact system with respect to a family $\mathcal{F}$ and $\mathcal{F}$ has FIP. When is $\mathcal{F}$ a filter, or contains a nontrivial filter?

Especially we address this question to the family $\mathcal{S}_{T}(\delta)$. More precisely, when a system $(X, T)$ is dynamically compact with respect to the family $\mathcal{N}_{T}$ and $\mathcal{N}_{T}$ has FIP, then, as well known, the systems is weakly mixing and $\mathcal{N}_{T}$ is a filter. Now, let a system $(X, T)$ is dynamically compact with respect to the family $\mathcal{S}_{T}(\delta)$ for some 
$\delta>0$ and $\mathcal{S}_{T}(\delta)$ has FIP, then the systems is multi-sensitive. But, the following question is still open - when is $\mathcal{S}_{T}(\delta)$ a filter?

A collection $\mathcal{H} \subset \mathcal{F}$ will be called a base for $\mathcal{F}$ if for any $F \in \mathcal{F}$ there is $H \in \mathcal{H}$ with $H \subset F$. We are interested in those families which have a countable base, that is, there exists a base $\mathcal{H}$ which is countable.

Note that not every Furstenberg family $\mathcal{F}$ has a countable base, for example, the family $\mathcal{B}$. Assume the contrary that $\mathcal{B}$ admits a countable base $\left\{F_{n}: n \in \mathbb{N}\right\}$. We take $k_{1} \in F_{1}$, and once $k_{m} \in F_{m}, m \in \mathbb{N}$ is defined we choose $k_{m+1} \in F_{m+1}$ with $k_{m+1}>k_{m}+m+1$. Set $E=\left\{k_{n}: n \in \mathbb{N}\right\}$ and $F=\mathbb{Z}_{+} \backslash E$. Then $E \cap F_{n} \neq \varnothing$ for all $n \in \mathbb{N}$, and $F \supset\left\{k_{m}+m: m \in \mathbb{N}\right\}$ and hence $F \in \mathcal{B}$, in particular, there exists no $n \in \mathbb{N}$ with $F_{n} \subset F$, a contradiction.

It is not hard to show even the existence of a family with FIP, but without a countable base. Nevertheless the families $\mathcal{N}_{T}$ and $\mathcal{S}_{T}(\delta)$ have countable bases. Indeed, we can consider a countable base $\mathcal{U}$ of open sets for the space $X$. Note that $U_{1} \subset U, V_{1} \subset V$ implies $N_{T}\left(U_{1}, V_{1}\right) \subset N_{T}(U, V)$ and $S_{T}\left(U_{1}, \delta\right) \subset S_{T}(U, \delta)$. Then $\left\{N_{T}(U, V): U, V \in \mathcal{U}\right\}$ and $\left\{S_{T}(U, \delta): U \in \mathcal{U}\right\}$ are countable bases for $\mathcal{N}_{T}$ and $\mathcal{S}_{T}(\delta)$, respectively.

The following is a general result that will be especially useful for families with countable bases.

Proposition 3.2. Let $(X, T)$ be a dynamical system and let $\mathcal{F}$ be a family such that there exists $x \in \operatorname{Tran}_{\mathcal{F}}(X, T)$. Then $\operatorname{orb}_{T}(x) \subset \operatorname{Tran}_{\mathcal{F}}(X, T)$.

Proof. By assumption, given an arbitrary opene $U \subset X$ we have that $N_{T}(x, U) \in \mathcal{F}$. Thus, for any $m \in \mathbb{N}$,

$$
N_{T}\left(T^{m} x, U\right)=N_{T}\left(x, T^{-m}(U)\right) \in \mathcal{F},
$$

and we conclude that $T^{m} x \in \operatorname{Tran}_{\mathcal{F}}(X, T)$.

Proposition 3.3. Assume that $\mathcal{F}$ admits a countable base $\mathcal{H}$. Then $\operatorname{Tran}_{k \mathcal{F}}(X, T)$ is a $G_{\delta}$ subset of $X$. Moreover, the following are equivalent:

(1) The system $(X, T)$ is $k \mathcal{F}$-transitive,

(2) $\operatorname{Tran}_{k \mathcal{F}}(X, T)$ is a dense $G_{\delta}$ subset of $X$,

(3) $\operatorname{Tran}_{k \mathcal{F}}(X, T) \neq \varnothing$.

Proof. Let $\mathcal{U}$ be a countable base of the family of all opene subsets of $X$. Then the class $\mathcal{U} \times \mathcal{H}$ is countable, and we enumerate it as $\left\{\left(U_{i}, F_{i}\right): i \in \mathbb{N}\right\}$. Denote by $T^{-F} U=\bigcup_{n \in F} T^{-n} U$ for any $F \subset \mathbb{Z}_{+}$and each $U \subset X$. Then it is easy to obtain

$$
\operatorname{Tran}_{k \mathcal{F}}(X, T)=\bigcap_{i=1}^{\infty} T^{-F_{i}} U_{i} .
$$

In fact, given arbitrary point $x \in X, x \in \operatorname{Tran}_{k \mathcal{F}}(X, T)$ if and only if $N_{T}(x, U) \in k \mathcal{F}$ for any opene subset $U$ of $X$, if and only if $N_{T}(x, U) \cap F \neq \varnothing$ for any opene subset $U$ of $X$ and each $F \in \mathcal{F}$, if and only if $N_{T}\left(x, U_{i}\right) \cap F_{i} \neq \varnothing$ for each $i \in \mathbb{N}$ by the construction. In particular, $\operatorname{Tran}_{k \mathcal{F}}(X, T)$ is a $G_{\delta}$ subset of $X$.

Thus $(X, T)$ is $k \mathcal{F}$-transitive, if and only if for any $F \in \mathcal{F}$ and arbitrary opene subsets $U, V$ of $X$ we have $N_{T}(V, U) \cap F \neq \varnothing$ and equivalently $T^{-F} U \cap V \neq \varnothing$, if and only if $T^{-F} U$ is an opene dense subset of $X$ for any $F \in \mathcal{F}$ and each opene subset $U$ of $X$, if and only if $\operatorname{Tran}_{k \mathcal{F}}(X, T)$ is a dense $G_{\delta}$ subset of $X$ by (3.1). 
Now we assume $\operatorname{Tran}_{k \mathcal{F}}(X, T) \neq \varnothing$. Let $x \in \operatorname{Tran}_{k \mathcal{F}}(X, T)$. By Proposition 3.2 $\operatorname{orb}_{T}(x) \subset \operatorname{Tran}_{k \mathcal{F}}(X, T)$, and hence $\operatorname{Tran}_{k \mathcal{F}}(X, T)$ is a dense $G_{\delta}$ subset of $X$ since $x \in \operatorname{Tran}(X, T)$. This finishes the proof.

Remark 3.4. Observe that when the state space $X$ is a compact metric space without isolated points, $x \in \operatorname{Tran}(X, T)$ if and only if $x \in \operatorname{Tran}_{\mathcal{B}}(X, T)$. The family $\mathcal{F}_{\text {cof }}$ is clearly translation invariant (and hence positively invariant) and admits a countable base, and $k \mathcal{F}_{\text {cof }}=\mathcal{B}$. Thus by Proposition 3.3 one has: $(X, T)$ is transitive if and only if $\operatorname{Tran}(X, T)$ is a dense $G_{\delta}$ subset of $X$ if and only if $\operatorname{Tran}(X, T) \neq \varnothing$.

\section{Transitive SENSITIVITY AND SENSITIVE COMPACTNESS}

Recall that a dynamical system $(X, T)$ is transitively sensitive if there exists $\delta>0$ such that $S_{T}(W, \delta) \cap N_{T}(U, V) \neq \varnothing$ for any opene subsets $U, V, W$ of $X$; and sensitive compact if there exists $\delta>0$ such that for any point $x \in X$ the set $\omega_{\mathcal{S}_{T}(\delta)}(x)$ is nonempty. Sometimes in that cases we will say also $(X, T)$ is transitively sensitive with a sensitive constant $\delta$ and $(X, T)$ is sensitive compact with a sensitive constant $\delta$. The main result of this section is the following

Theorem 4.1. Let $(X, T)$ be a minimal system. Then the following conditions are equivalent:

(1) $(X, T)$ is multi-sensitive.

(2) $(X, T)$ is sensitive compact.

(3) There exists $\delta>0$ such that $\omega_{\mathcal{S}_{T}(\delta)}(x)=X$ for each $x \in X$.

(4) There exist $\delta>0$ and $x \in X$ with $\omega_{\mathcal{S}_{T}(\delta)}(x)=X$.

(5) $(X, T)$ is transitively sensitive.

Before proceeding, we need:

Lemma 4.2. Let $\delta>0$ and $x \in X$. If $T: X \rightarrow X$ is almost open, then the family $\mathcal{S}_{T}(\delta)$ is negatively invariant, and the subset $\omega_{\mathcal{S}_{T}(\delta)}(x)$ is positively $T$-invariant.

Proof. By Proposition 2.1 it suffices to prove that $\mathcal{S}_{T}(\delta)$ is a negatively invariant family. Take arbitrary $F \in \mathcal{S}_{T}(\delta)$ and any $i \in \mathbb{Z}_{+}$. Then there exists opene subset $U$ of $X$ with $S_{T}(U, \delta) \subset F$. As $T: X \rightarrow X$ is almost open, $T^{i}: X \rightarrow X$ is also almost open, and then we can choose opene $V \subset T^{i} U$. One has $g^{-i}(F) \supset g^{-i} S_{T}(U, \delta)=$ $S_{T}\left(T^{i} U, \delta\right) \supset S_{T}(V, \delta)$, which implies that the family $\mathcal{S}_{T}(\delta)$ is negatively invariant.

The following result gives a characterization of transitive sensitivity for a general dynamical system in terms of dynamical compactness.

Proposition 4.3. Let $(X, T)$ be a dynamical system. Then the family $\mathcal{S}_{T}(\delta)$ is positively invariant for any $\delta>0$. Furthermore, the following conditions are equivalent:

(1) $(X, T)$ is transitively sensitive.

(2) There exist a $\delta>0$ and a dense $G_{\delta}$ subset $X_{0} \subset X$ such that $\omega_{\mathcal{S}_{T}(\delta)}(x)=X$ for each $x \in X_{0}$.

(3) There exist $a \delta>0$ and a point $x \in X$ with $\omega_{\mathcal{S}_{T}(\delta)}(x)=X$. 
Proof. Firstly, we show that $\mathcal{S}_{T}(\delta)$ is a positively invariant family. In fact, take any $F \in \mathcal{S}_{T}(\delta)$ and any $i \in \mathbb{Z}_{+}$. We choose opene subsets $U, V$ of $X$ with $F \supset S_{T}(U, \delta)$ and $V \subset T^{-i} U$ satisfying $\operatorname{diam}\left(T^{j} V\right)<\delta$ for all $j=0,1, \ldots, i$. Thus $g^{i}(F) \supset$ $g^{i} S_{T}(U, \delta) \supset S_{T}(V, \delta)$ from the construction, and then $g^{i}(F) \in \mathcal{S}_{T}(\delta)$. This implies the positive invariance of the family $\mathcal{S}_{T}(\delta)$.

Observe that $(X, T)$ is transitively sensitive with a sensitive constant $\delta$, if and only if $(X, T)$ is $k \mathcal{S}_{T}(\delta)$-transitive; and that the family $\mathcal{S}_{T}(\delta)$ has a countable base: let $\mathcal{U}$ be a countable base of the family of all opene subsets of $X$, then $\left\{S_{T}(U, \delta)\right.$ : $U \in \mathcal{U}\}$ is a countable base of $\mathcal{S}_{T}(\delta)$. Then applying Proposition 3.3 the equivalence of $(1) \Leftrightarrow(2) \Leftrightarrow(3)$ follows from the fact that $x \in \operatorname{Tran}_{k \mathcal{S}_{T}(\delta)}(X, T)$ if and only if $\omega_{\mathcal{S}_{T}(\delta)}(x)=X$.

We recall that by [17, Corollary 1.7] the sensitivity of a dynamical system can be lifted up from a factor to an extension by an almost open factor map between transitive systems. The following result shows that the transitive sensitivity can be lifted up to an extension from a factor by an almost one-to-one factor map and that the transitive sensitivity is projected from an extension to the sensitivity of a factor by a weakly almost one-to-one factor map.

Lemma 4.4. Let $\pi:(X, T) \rightarrow(Z, R)$ be a factor map between dynamical systems.

(1) Assume that $\pi$ is almost one-to-one. If $(Z, R)$ is transitively sensitive with a sensitive constant $\delta>0$ then $(X, T)$ is also transitively sensitive.

(2) Assume that there exists $z \in Z$ whose fiber is a singleton. If $(X, T)$ is transitively sensitive then $(Z, R)$ is sensitive, in particular, $E q(Z, R)=\varnothing$.

Proof. (1) We take a compatible metric $\rho$ over $Z$ and let $\varepsilon>0$ such that $d\left(x_{1}, x_{2}\right) \leq$ $\varepsilon$ implies $\rho\left(\pi x_{1}, \pi x_{2}\right) \leq \delta$ for any $x_{1}, x_{2} \in X$. Now let $U, V, W$ be arbitrary opene subsets of $X$. As the map $\pi: X \rightarrow Z$ is almost one-to-one, we may take opene subsets $U_{Z}, V_{Z}, W_{Z}$ of $Z$ with $\pi^{-1} U_{Z} \subset U, \pi^{-1} V_{Z} \subset V$ and $\pi^{-1} W_{Z} \subset W$. Observe that if $n \in S_{R}\left(W_{Z}, \delta\right) \cap N_{R}\left(U_{Z}, V_{Z}\right)$, then: on one hand, there exist $z_{1}, z_{2} \in W_{Z}$ with $\rho\left(R^{n} z_{1}, R^{n} z_{2}\right)>\delta$, and so $d\left(T^{n} x_{1}, T^{n} x_{2}\right)>\varepsilon$ for any $x_{1} \in \pi^{-1}\left(z_{1}\right)$ and $x_{2} \in \pi^{-1}\left(z_{2}\right)$, hence $\operatorname{diam}\left(T^{n} W\right)>\varepsilon$; on the other hand, $U_{Z} \cap R^{-n} V_{Z} \neq \varnothing$, and then $U \cap S^{-n} V \supset \pi^{-1} U_{Z} \cap \pi^{-1}\left(R^{-n} V_{Z}\right) \neq \varnothing$. This implies $S_{T}(W, \varepsilon) \cap N_{T}(U, V) \supset$ $S_{R}\left(W_{Z}, \delta\right) \cap N_{R}\left(U_{Z}, V_{Z}\right) \neq \varnothing$, as $(Z, R)$ is transitively sensitive. Thus, by the arbitrariness of $U, V$ and $W$, we have that $(X, T)$ is also transitively sensitive.

(2) As $(X, T)$ is transitively sensitive (with a sensitive constant $\delta>0$ ), it is clear that $(Z, R)$ is transitive, and then by the refined Auslander-York dichotomy the system $(Z, R)$ is sensitive if and only if $\operatorname{Eq}(Z, R)=\varnothing$ (see [6], [17], [2] and the book [1]). Thus it suffices to prove $\operatorname{Eq}(Z, R)=\varnothing$. Assume to the contrary that $\operatorname{Eq}(Z, R)$ is a nonempty set. Let $\rho$ be a compatible metric over $Z$, and take a point $z \in \operatorname{Eq}(Z, R)$. By the assumption that there exists a point of $Z$ whose fiber is a singleton, we may take an opene subset $W$ in $Z$ with $\operatorname{diam}\left(\pi^{-1} W\right)<\delta$, and an opene subset $W_{*} \subset W$ and $\delta_{*}>0$ such that if the distance between a point of $Z$ and $W_{*}$ is smaller than $\delta_{*}$ then the point belongs to $W$. Since $z \in$ $\operatorname{Eq}(Z, R)$, there exists an open neighborhood $U_{*}$ of $z$ with $\operatorname{diam}\left(R^{n} U_{*}\right)<\delta_{*}$ for all $n \in \mathbb{Z}_{+}$. As $(X, T)$ is transitively sensitive with a sensitive constant $\delta$, take $m \in N_{T}\left(\pi^{-1} U_{*}, \pi^{-1} W_{*}\right) \cap S_{T}\left(\pi^{-1} U_{*}, \delta\right)$. Thus $T^{m}\left(\pi^{-1} U_{*}\right) \cap \pi^{-1} W_{*} \neq \varnothing$, and then $R^{m} U_{*} \cap W^{*} \neq \varnothing$, which implies $R^{m} U_{*} \subset W$ by the construction of $U_{*}, W_{*}$ and $W$. Hence

$$
\operatorname{diam}\left(T^{m}\left(\pi^{-1} U_{*}\right)\right) \leq \operatorname{diam}\left(\pi^{-1}\left(R^{m} U_{*}\right)\right) \leq \operatorname{diam}\left(\pi^{-1} W\right)<\delta,
$$


a contradiction to the selection of $m \in S_{T}\left(\pi^{-1} U_{*}, \delta\right)$. This finishes the proof.

Now we are ready to prove Theorem 4.1.

Proof of Theorem 4.1. (1) $\Rightarrow(2)$ follows directly from the definitions. As the system $(X, T)$ is minimal, the map $T: X \rightarrow X$ is almost open. Observing that $\omega_{\mathcal{S}_{T}(\delta)}(x)$ is a closed subset of $X$ for each $x \in X$, the implication of $(2) \Rightarrow(3)$ follows from Lemma 4.2 and the minimality of $(X, T)$. The implication of $(3) \Rightarrow(4) \Rightarrow(5)$ follows from Proposition 4.3. Since a minimal system is either multi-sensitive or a weakly almost one-to-one extension of its maximal equicontinuous factor by [24], then $(5) \Rightarrow(1)$ follows from Lemma 4.4. This finishes the proof.

Clearly each multi-sensitive system is sensitive compact. Observe that each non-proximal, transitive compact system is multi-sensitive by [23, Theorem 4.7]. In particular, each minimal transitive compact system is multi-sensitive, as each minimal proximal system is trivial by [4] and all dynamical systems considered are assumed to be nontrivial. Nevertheless, there are many minimal, non transitive compact, multi-sensitive systems. For example, consider the classical dynamical system $(X, T)$ given by $X=\mathbb{R}^{2} / \mathbb{Z}^{2}$ and $T:(x, y) \mapsto(x+\alpha, x+y)$ with $\alpha \notin \mathbb{Q}$ (see $[15$, Chapter 1]). As commented in [23, Page 1816], $(X, T)$ is an invertible minimal multi-sensitive system; note that $(X, T)$ is not weakly mixing, since $(X, T)$ admits an irrational rotation as its nontrivial equicontinuous factor and any equicontinuous factor of a weakly mixing system is trivial. Recall that by [23, Corollary 3.10] for a minimal system the system is transitive compact if and only if it is weakly mixing, and then the constructed system $(X, T)$ is not transitive compact.

Proposition 4.5. Each nontrivial weakly mixing system $(X, T)$ is transitively sensitive.

Proof. As the system $(X, T)$ is nontrivial, we take $0<\delta<\operatorname{diam}(X)$. Choose opene subsets $W_{1}, W_{2}$ of $X$ such that the distance between $W_{1}$ and $W_{2}$ is strictly larger than $\delta$. Now take arbitrary opene subsets $U, V, W$ of $X$. As $(X, T)$ is weakly mixing, $\left(X^{3}, T^{(3)}\right)$ is transitive by [14], and then $N_{T}(U, V) \cap S_{T}(W, \delta) \supset N_{T^{(3)}}(U \times$ $\left.W \times W, V \times W_{1} \times W_{2}\right) \neq \varnothing$. This implies that $(X, T)$ is transitively sensitive with a sensitive constant $\delta>0$.

We give a sufficient condition for a dynamical system being transitively sensitive (by Proposition 4.3) as the last result of this section.

Lemma 4.6. Assume $\omega_{\mathcal{S}_{T}(\varepsilon)}(x)=X$ for some $x \in X$ and $\varepsilon>0$. Then there is $\delta>0$ such that for any opene subset $U$ of $X$ and each neighbourhood $U_{x}$ of $x$ there are $y \in U_{x}$ and $n \in N_{T}(x, U)$ with $d\left(T^{n} x, T^{n} y\right)>\delta$. If in addition, the map $T: X \rightarrow X$ is almost one-to-one, then the converse holds.

Proof. Fix an opene subset $U$ of $X$ and a neighborhood $U_{x}$ of $x$. As $\omega_{\mathcal{S}_{T}(\varepsilon)}(x)=X$, there is $n \in N_{T}(x, U) \cap S_{T}\left(U_{x}, \varepsilon\right)$, and then there are points $x_{1}, x_{2} \in U_{x}$ with $d\left(T^{n} x_{1}, T^{n} x_{2}\right)>\varepsilon$. We have either $d\left(T^{n} x, T^{n} x_{1}\right)>\frac{\varepsilon}{2}$ or $d\left(T^{n} x, T^{n} x_{2}\right)>\frac{\varepsilon}{2}$, and then obtain the desired statement for $\delta=\frac{\varepsilon}{2}$.

Now suppose that there is $\delta>0$ such that for any opene subset $U$ of $X$ and each neighbourhood $U_{x}$ of $x$ there are $y \in U_{x}$ and $n \in N_{T}(x, U)$ with $d\left(T^{n} x, T^{n} y\right)>\delta$, and that the map $T: X \rightarrow X$ is almost one-to-one. Let $U, W$ be arbitrary opene subsets of $X$. It is clear that $x \in \operatorname{Tran}(X, T)$, and so there is $k \in N_{T}(x, W)$. Note that $T: X \rightarrow X$ is almost one-to-one, so the map $T^{k}: X \rightarrow X$ is also almost 
one-to-one by Corollary 2.4, and then we may take an opene subset $V$ of $X$ with $T^{-k} V=\left(T^{k}\right)^{-1} V \subset U$. Observe that $T^{-k} W$ is a neighborhood of $x$, and then by the assumption there exists a point $y \in T^{-k} W$ and an integer $n \in \mathbb{Z}_{+}$such that $n \in N_{T}(x, V)$ and $d\left(T^{n} x, T^{n} y\right)>\delta$. In fact, we may assume $n>k$, else we can replace $T^{-k} W$ by a small enough open neighbourhood $G_{x} \subset T^{-k} W$ of $x$ with $\operatorname{diam}\left(T^{i} G_{x}\right)<\delta$ for all $0 \leq i \leq k$. Then $\operatorname{diam}\left(T^{n-k} W\right)>\delta$ as $T^{k} x \in W$ and $T^{k} y \in$ $W$, and $T^{n-k} x \in T^{-k}\left(T^{n} x\right) \subset T^{-k} V \subset U$. In particular, $N_{T}(x, U) \cap S_{T}(W, \delta) \neq \varnothing$. Thus $\omega_{\mathcal{S}_{T}(\delta)}(x)=X$ by the arbitrariness of $U$ and $W$.

\section{WEAKLY DISJOINTNESS AND WEAKLY MIXING}

Recall that dynamical systems $(X, T)$ and $(Y, S)$ are weakly disjoint if the product system $(X \times Y, T \times S)$ is transitive. The following theorem characterizes weak disjointness, which is proved firstly by Weiss [39] in some special class and then is generalized by Akin and Glasner [3]. We say that $\mathcal{F}$ is thick if $\tau \mathcal{F}=\mathcal{F}$, where

$$
\tau \mathcal{F}=\left\{F \subset \mathbb{Z}_{+}: \bigcap_{j=1}^{n} g^{-i_{j}} F \in \mathcal{F} \text { for each } n \in \mathbb{N} \text { and all } i_{1}, \ldots, i_{n} \in \mathbb{Z}_{+}\right\} \text {. }
$$

Weiss-Akin-Glasner Theorem. Let $\mathcal{F}$ be a proper, translation invariant, thick family. A dynamical system is $k \mathcal{F}$-transitive if and only if it is weakly disjoint from every $\mathcal{F}$-transitive system.

Observe that a dynamical system is weakly mixing if and only if it is weakly disjoint from itself, and then weak disjointness is characterized by [23, Proposition 3.8] in some special case. Now we discuss weak disjointness using dynamical compactness which will be some generalization of [23, Proposition 3.8]. We will need the following

Lemma 5.1. Let $(X, T)$ and $(Y, S)$ be dynamical systems and let $x \in X$. Then the family $\mathcal{N}_{S}$ is translation invariant and $\omega_{\mathcal{N}_{S}}(x)=\omega_{\mathcal{N}_{S}}(T x)$.

Proof. By Proposition 2.1 it suffices to prove that $\mathcal{N}_{S}$ is a translation invariant family. We also suppose that $\mathcal{N}_{S}$ is proper (i.e., $(Y, S)$ is a transitive system) since, otherwise, the result is trivial. Take arbitrary $F \in \mathcal{N}_{S}$ and any $i \in \mathbb{Z}_{+}$. Then there exist opene subsets $U, V$ of $Y$ with $N_{S}(U, V) \subset F$. As the non-singleton space $Y$ contains no isolated points, we can take suitable opene $V_{1} \subset V$ and $U_{1} \subset U$ such that $U_{1} \cap \bigcup_{k=0}^{i} S^{k} \overline{V_{1}}=\varnothing$. One has $g^{i} N_{S}(U, V) \supset N_{S}\left(S^{-i} U_{1}, V_{1}\right)$, which implies that the family $\mathcal{N}_{S}$ is positively invariant: in fact, if $n \in N_{S}\left(S^{-i} U_{1}, V_{1}\right)$ then $n>i$ by the selection, and so $n-i \in N_{S}\left(U_{1}, V_{1}\right) \subset N_{S}(U, V)$. Moreover, it is clear $g^{-i}(F) \supset g^{-i} N_{S}(U, V) \supset N_{S}\left(U, S^{-i} V\right)$, and then the family $\mathcal{N}_{S}$ is negatively invariant. This finishes the proof.

Theorem 5.2. The following conditions are equivalent:

(1) The systems $(X, T)$ and $(Y, S)$ are weakly disjoint.

(2) Both $\operatorname{Tran}_{k \mathcal{N}_{S}}(X, T)$ and $\operatorname{Tran}_{k \mathcal{N}_{T}}(Y, S)$ are dense $G_{\delta}$ subsets.

(3) The set $\operatorname{Tran}_{k \mathcal{N}_{S}}(X, T)$ is a dense $G_{\delta}$ subset of $X$.

(4) Both $\operatorname{Tran}_{k \mathcal{N}_{S}}(X, T)$ and $\operatorname{Tran}_{k \mathcal{N}_{T}}(Y, S)$ are nonempty subsets.

(5) The set $\operatorname{Tran}_{k \mathcal{N}_{S}}(X, T)$ is a nonempty subset of $X$.

Proof. $(1) \Leftrightarrow(2) \Leftrightarrow(3)$ : It is clear from the definition that: the system $(X, T)$ is $k \mathcal{N}_{S}$-transitive, if and only if the systems $(X, T)$ and $(Y, S)$ are weakly disjoint, if 
and only if $(X, T)$ is $k \mathcal{N}_{S}$-transitive and $(Y, S)$ is $k \mathcal{N}_{T}$-transitive. As both $\mathcal{N}_{T}$ and $\mathcal{N}_{S}$ are families admitting a countable base, it is direct to obtain the equivalence of $(1) \Leftrightarrow(2) \Leftrightarrow(3)$ by applying Proposition 3.3 .

The implication $(2) \Rightarrow(4) \Rightarrow(5)$ is obvious. To finish the proof, we only need to show $(5) \Leftrightarrow(3)$. By Lemma 5.1 the family $\mathcal{N}_{S}$ is translation invariant, and then the family $k \mathcal{N}_{S}$ is also translation invariant. Thus the equivalence of $(5) \Leftrightarrow(3)$ follows from Proposition 3.3.

Note that we have a characterization of weak mixing by using dynamical compactness [23, Proposition 3.8]. Now we extend [23, Proposition 3.8] as follows.

Recall that $\mathcal{S} \subset \mathbb{N}$ is an IP set if there exists $\left\{p_{k}: k \in \mathbb{N}\right\} \subset \mathbb{N}$ with $F S\left\{p_{i}\right\}_{i=1}^{\infty} \subset$ $\mathcal{S}$, where $F S\left\{p_{i}\right\}_{i=1}^{\infty}=\left\{p_{i_{1}}+\cdots+p_{i_{k}}: k \in \mathbb{N}\right.$ and $\left.1 \leq i_{1}<\cdots<i_{k}\right\}$. Analogously, for each $n \in \mathbb{N}$ we define $F S\left\{p_{i}\right\}_{i=1}^{n}=\left\{p_{i_{1}}+\cdots+p_{i_{k}}: k \in \mathbb{N}\right.$ and $1 \leq i_{1}<\cdots<$ $\left.i_{k} \leq n\right\}$. Denote by $\mathcal{F}_{i p}$ the family of all IP sets.

By [28, Theorem 3.2], the subset $\operatorname{Tran}_{\mathcal{F}_{i p}}(X, T)$ contains a dense $G_{\delta}$ subset of $X$ for any weakly mixing system $(X, T)$, while $\operatorname{Tran}_{\mathcal{F}_{i p}}(X, T) \neq \varnothing$ does not imply the weak mixing of the system $(X, T)$ by $[28$, Proposition 3.4]. We will extend that in the following Proposition 5.4. Before proceeding, we make the following

Lemma 5.3. Let $(X, T)$ be a dynamical system and $\mathcal{F}$ be a family.

(1) Let $\delta>0$. If the family $\mathcal{S}_{T}(\delta) \cdot \mathcal{F}$ is proper then $\mathcal{S}_{T}(\delta) \cdot \mathcal{F} \subset \mathcal{B}$.

(2) If the family $\mathcal{N}_{T} \cdot \mathcal{F}$ is proper then $\mathcal{N}_{T} \cdot \mathcal{F} \subset \mathcal{B}$.

Proof. (1) Assume the contrary that there exists an opene subset $U$ in $X$ and $F \in \mathcal{F}$ such that $S_{T}(U, \delta) \cap F$ is finite, and so we may choose $m \in \mathbb{N}$ such that $n \notin S_{T}(U, \delta) \cap F$ for any integer $n>m$. Since $T: X \rightarrow X$ is uniformly continuous one can find opene $V \subset U$ small enough such that $\operatorname{diam}\left(T^{i} V\right)<\delta$ for all $0 \leq i \leq m$. Then $S_{T}(V, \delta) \subset S_{T}(U, \delta)$, which implies $S_{T}(V, \delta) \cap F=\varnothing$. A contradiction.

(2) Assume the contrary that there exist opene subsets $U, V$ in $X$ and $F \in \mathcal{F}$ such that $N_{T}(U, V) \cap F$ is finite, say $N_{T}(U, V) \cap F=\left\{n_{1}, \ldots, n_{k}\right\}$. As the non-singleton space $X$ contains no isolated points, we can take opene $U_{1} \subset U$ small enough such that $V_{1}:=V \backslash \bigcup_{i=1}^{k} T^{n_{i}} \overline{U_{1}}$ is an opene subset of $X$. By the construction we have $N_{T}\left(U_{1}, V_{1}\right) \subset N_{T}(U, V)$ and then $N_{T}\left(U_{1}, V_{1}\right) \cap F=\varnothing$, a contradiction.

Proposition 5.4. The following conditions are equivalent:

(1) $(X, T)$ is weakly mixing.

(2) There exists a dense $G_{\delta}$ subset $X^{\prime}$ of $X$ such that, for each $x \in X^{\prime}$, $N_{T}(x, G) \cap N_{T}(U, V) \in \mathcal{F}_{i p}$ for all opene subsets $G, U, V$ of $X$.

Proof. $(2) \Rightarrow(1)$ : Just observe from the assumption that $\omega_{\mathcal{N}_{T}}(x)=X$ for all $x \in X^{\prime}$, and hence the system $(X, T)$ is weakly mixing by [23, Proposition 3.8].

$(1) \Rightarrow(2)$ : Since $(X, T)$ is weakly mixing, $\left(X^{2}, T^{(2)}\right)$ is also weakly mixing by [14] and [35], and hence by [23, Proposition 3.8] there is a dense $G_{\delta}$ subset $Y \subset X^{2}$ such that $\omega_{\mathcal{N}_{T(2)}}\left(\left(x_{1}, x_{2}\right)\right)=X^{2}$ for each $\left(x_{1}, x_{2}\right) \in Y$. Applying the well-known Ulam Lemma there is a dense $G_{\delta}$ subset $X^{\prime} \subset X$ such that, for any $x \in X^{\prime}$, $\{y:(x, y) \in Y\}$ is a dense $G_{\delta}$ subset of $X$. Now we show that $X^{\prime}$ is the desired set.

Let $x \in X^{\prime}$ and fix any opene subsets $G, U, V$ of $X$. Choose $y \in G$ with $(x, y) \in Y$ and then $\omega_{\mathcal{N}_{T^{(2)}}}((x, y))=X^{2}$, in particular, $(y, y) \in \omega_{\mathcal{N}_{T(2)}}((x, y))$. Thus

$$
N_{T}(x, G) \cap N_{T}(y, G) \cap N_{T}(U, V) \cap N_{T}(V, V) \neq \varnothing,
$$


and take $p_{1} \in \mathbb{N}$ from this set by Lemma 5.3. We have $p_{1} \in N_{T}(x, G) \cap N_{T}(U, V)$ and $T^{p_{1}} y \in G, T^{p_{1}} V \cap V \neq \varnothing$. Define opene subsets $G_{1}=G \cap T^{-p_{1}} G \ni y$ and $V_{1}=V \cap T^{-p_{1}} V$. Now we proceed inductively. Suppose that we are given a sequence $\left\{p_{1}, \ldots, p_{k}\right\} \subset \mathbb{N}$ with $F S\left\{p_{i}\right\}_{i=1}^{k} \subset N_{T}(x, G) \cap N_{T}(U, V)$, and opene subsets

$$
G_{k}=\bigcap_{s \in F S\left\{p_{i}\right\}_{i=1}^{k} \cup\{0\}} T^{-s} G \ni y, V_{k}=\bigcap_{s \in F S\left\{p_{i}\right\}_{i=1}^{k} \cup\{0\}} T^{-s} V .
$$

As $(y, y) \in \omega_{\mathcal{N}_{T^{(2)}}}((x, y))$, we may take $p_{k+1} \in \mathbb{N}$ by Lemma 5.3 from the set $N_{T}\left(x, G_{k}\right) \cap N_{T}\left(y, G_{k}\right) \cap N_{T}\left(U, V_{k}\right) \cap N_{T}\left(V_{k}, V_{k}\right)$. It is not hard to check that

$$
\begin{gathered}
G_{k+1}=G_{k} \cap T^{-p_{k+1}} G_{k}=\bigcap_{s \in F S\left\{p_{i}\right\}_{i=1}^{k+1} \cup\{0\}} T^{-s} G \ni y, \\
V_{k+1}=V_{k} \cap T^{-p_{k+1}} V_{k}=\bigcap_{s \in F S\left\{p_{i}\right\}_{i=1}^{k+1} \cup\{0\}} T^{-s} V
\end{gathered}
$$

are both opene subsets of $X$, and that $F S\left\{p_{i}\right\}_{i=1}^{k+1} \subset N_{T}(x, G) \cap N_{T}(U, V)$, which completes the induction. Finally, $N_{T}(x, G) \cap N_{T}(U, V) \in \mathcal{F}_{i p}$ with $F S\left\{p_{i}\right\}_{i=1}^{\infty} \subset$ $N_{T}(x, G) \cap N_{T}(U, V)$. This finishes the proof.

\section{TRANSITIVE COMPACT (NON WEAKLY MIXING) SYSTEMS}

Recall that the system $(X, T)$ is totally transitive if $\left(X, T^{k}\right)$ is transitive for each $k \in \mathbb{N}$; and is topologically mixing if $N_{T}(U, V) \in \mathcal{F}_{\text {cof }}$ for any opene subsets $U, V$ in $X$. Note that $(X, T)$ is weakly mixing if and only if $N_{T}(U, V) \in \mathcal{F}_{\text {thick }}$ for any opene sets $U, V$ in $X$ by $[14,35]$, and so any weakly mixing system is totally transitive. It is direct to check that each weakly mixing system is transitive compact. In [23] the authors showed the existence of non totally transitive, transitive compact systems in both proximal and non-proximal cases. We extend it as follows:

Theorem 6.1. There is a totally transitive, transitive compact system $(X, T)$ which is not weakly mixing.

Proof. Let $\left(\mathbb{S}^{1}, R_{\alpha}\right)$ be the standard irrational rotation on the unit circle $\mathbb{S}^{1}=\mathbb{R} / \mathbb{Z}$ with $\alpha \notin \mathbb{Q}$. Take a nontrivial proximal, topologically mixing system $(Y, S)$ (for the existence of such a system see some example [22]). Note that a dynamical system is proximal if and only if it contains the unique fixed point, which is the only minimal point of the system [4]. Denote by $p_{Y}$ the unique minimal point (fixed point) of $(Y, S)$. Observe that the system $\left(Y \times \mathbb{S}^{1}, S \times R_{\alpha}\right)$ is totally transitive: for each $n \in \mathbb{N}$, the system $\left(Y, S^{n}\right)$ is topologically mixing by the definition and it is standard that the system $\left(\mathbb{S}^{1}, R_{\alpha}^{n}\right)$ is minimal, then it is direct to see that these two systems are weakly disjoint.

Let $(X, T)$ be the quotient system $Y \times \mathbb{S}^{1} / \sim$ equipped with the action $T$ induced naturally from $S \times R_{\alpha}$, where the equivalence relation $\sim$ is defined via: given $x, y \in X, x \sim y$ if and only if either $x=y$ or $x$ and $y$ both have $p_{Y}$ in the first coordinate. In other words the space $X$ looks like a cone space, where the vertex of the cone is a point $p$, each "horizontal" fiber spaces are the space $Y$, the vertical fiber spaces are the circles (see Figure 1). Clearly, $(X, T)$ is totally transitive. 


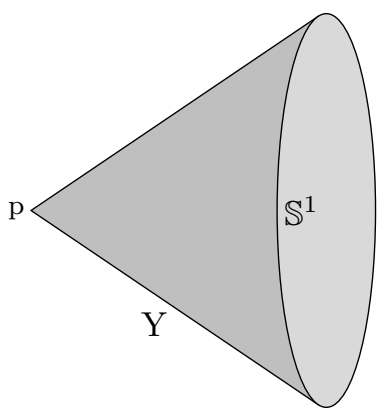

Figure 1.

Denote by $q: Y \times \mathbb{S}^{1} \rightarrow X$ the corresponding quotient map, then $q: Y_{\infty} \times \mathbb{S}^{1} \rightarrow$ $X \backslash\{p\}$ is a homeomorphism, where we set $Y_{\infty}=Y \backslash\left\{p_{Y}\right\}$. It is standard that the system $\left(\mathbb{S}^{1}, R_{\alpha}\right)$ is not weakly mixing, and then there exist opene subsets $U_{*}, V_{*}$ of $\mathbb{S}^{1}$ with $N_{R_{\alpha}}\left(U_{*}, V_{*}\right) \notin \mathcal{F}_{\text {thick}}$, hence

$$
N_{T}\left(q\left(Y_{\infty} \times U_{*}\right), q\left(Y_{\infty} \times V_{*}\right)\right)=N_{S \times R_{\alpha}}\left(Y_{\infty} \times U_{*}, Y_{\infty} \times V_{*}\right) \subset N_{R_{\alpha}}\left(U_{*}, V_{*}\right)
$$

is not thick. This implies that the system $(X, T)$ is not weakly mixing.

Now let $U, V$ be arbitrary opene subsets of $X$. We can choose opene subsets $U_{1}, V_{1} \subset Y_{\infty}$ and $U_{2}, V_{2} \subset \mathbb{S}^{1}$ with $U_{1} \times U_{2} \subset q^{-1} U$ and $V_{1} \times V_{2} \subset q^{-1} V$. As $(Y, S)$ is topologically mixing and $\left(\mathbb{S}^{1}, R_{\alpha}\right)$ is minimal, $N_{S}\left(U_{1}, V_{1}\right) \in \mathcal{F}_{\text {cof }}$ and $N_{R_{\alpha}}\left(U_{2}, V_{2}\right) \in$ $\mathcal{F}_{\text {syn }}$, and then $N_{S}\left(U_{1}, V_{1}\right) \cap N_{R_{\alpha}}\left(U_{2}, V_{2}\right) \in \mathcal{F}_{\text {syn }}$, thus

$$
N_{T}(U, V)=N_{S \times R_{\alpha}}\left(q^{-1} U, q^{-1} V\right) \supset N_{S \times R_{\alpha}}\left(U_{1} \times U_{2}, V_{1} \times V_{2}\right)
$$

is a syndetic set. Observe from the construction that the system $(X, T)$ is proximal with $p$ as its unique fixed point, then $N_{T}\left(x, U_{p}\right)$ is a thickly syndetic subset for each point $x \in X$ and any neighbourhood $U_{p}$ of $p$ (see [23, Lemma 3.12]). This implies $p \in \omega_{\mathcal{N}_{T}}(x)$ for each $x \in X$, and then the system $(X, T)$ is transitive compact.

The following result is proved independently in [11] and [38].

Lemma 6.2. Any $\omega$-limit set $\omega_{T}(x)$ can not be decomposed into $\alpha$ disjoint closed, nonempty, positively $T$-invariant subsets, where $2 \leq \alpha \leq \aleph_{0}$.

Before proceeding, we need the following example, for which we fail to find a reference and hence provide a detailed construction, as it is crucial in our arguments.

Proposition 6.3. For any given compact metric space $Z$, there exists a topologically mixing system $(X, T)$ such that, $Z$ can be realized as the set of all of its minimal points, furthermore, its each minimal point is a fixed point.

Proof. The construction is divided into two steps.

In the first step we shall construct a topologically mixing system $(Y, F)$ but with two fixed points, which are the only minimal points of the system. Let $\Sigma=\{0,1\}^{\mathbb{Z}_{+}}$ and $\sigma: \Sigma \rightarrow \Sigma$ be the full (one-sided) shift. We are going to find the system $(Y, F)$ of the form $\left(\overline{\operatorname{orb}_{\sigma}(x)}, \sigma\right)$ for some $x \in \Sigma$.

In order to define $x \in \Sigma$, firstly we represent each $W \in \Sigma^{*}$ with $|W| \geq 1$ in the form of $W=a^{i} Q b^{j}$ with $a, b \in\{0,1\}$ and $i \in \mathbb{N}, j \in \mathbb{Z}_{+}$as follows. Assume $W=w_{1} \ldots w_{|W|}$ with $w_{1}, \ldots, w_{|W|} \in\{0,1\}$. If $w_{1}=\cdots=w_{|W|}$ then we set $Q$ to be the empty subblock and $a=w_{1}, i=|W|, b=0, j=0$. If $w_{i} \neq w_{j}$ for some $1 \leq i<j \leq|W|$ then we set $a=w_{1}, b=w_{|W|}$ and choose $i, j \in \mathbb{N}$ such that $a^{i}$ and 
$b^{j}$ are the longest segments of equal digits which we can take at the beginning and at the end of $W$, respectively, whereas $Q$ is the rest, possible the empty subblock. In particular, if $W=a^{k}$ with $a \in\{0,1\}$ and $k \in \mathbb{N}$ then we set $i=k, b=0, j=0$.

Now starting with $A_{1}=10$, we construct inductively the blocks $A_{2}, A_{3}, \ldots$, and then define $x \in \Sigma$ as the limit of the starting blocks $A_{k}$. Suppose that we have defined $A_{k}, k \in \mathbb{N}$. Since $A_{k}$ has finitely many subblocks, there is a finite number of different pairs of these subblocks. For any pair $\left(W_{1}, W_{2}\right)$ of subblocks of $A_{k}$ we define a block $c\left(W_{1}, W_{2}\right)$ by using their combination as follows. The definition $c\left(W_{1}, W_{2}\right)$ depends on the structure of $W_{1}$ and $W_{2}$. Let us write $W_{1}$ and $W_{2}$ in the form as in the previous paragraph: $W_{1}=a^{i_{1}} Q_{1} b^{j_{1}}$ and $W_{2}=c^{i_{2}} Q_{2} d^{j_{2}}$ with $a, b, c, d \in\{0,1\}$ and $i_{1}, i_{2} \in \mathbb{N}, j_{1}, j_{2} \in \mathbb{Z}_{+}$, whereas $Q_{1}$ and $Q_{2}$ are possible empty subblocks. The combination block $c\left(W_{1}, W_{2}\right)$ of the pair $W_{1}, W_{2}$ is defined as

$$
c\left(W_{1}, W_{2}\right)=a^{k+i_{1}} Q_{1} b^{j_{1}+k} c^{k+i_{2}} Q_{2} d^{k+j_{2}} a^{k+i_{1}} Q_{1} b^{j_{1}+k+1} c^{k+i_{2}} Q_{2} d^{k+j_{2}} .
$$

Now let us define $A_{k+1}$ : at the beginning of $A_{k+1}$ we write $A_{k} 0^{k} 1^{k}$, and then all possible blocks $c\left(W_{1}, W_{2}\right)$ of pairs $\left(W_{1}, W_{2}\right)$ of subbloks of $A_{k}$ in any fixed order.

We see from the construction that $A_{k+1}$ is presented as a sequence of blocks with length not longer than $\left|A_{k}\right|$, which are separated from each other with some sequences of blocks of consecutive 1's or 0's of length not less than $k$. In fact, for all $m>k$ this property holds for $A_{m}$ (and hence in any subblock of $A_{m}$ with length more than $\left|A_{k}\right|+2 k-1$ one can find $0^{k}$ or $1^{k}$ ). Suppose that $A_{m}$ may be presented in this form. Since a block $W$ is presented as $a^{i} Q b^{j}$ and the property holds for $W$, it holds for $Q$. Indeed, if $i \geq k$ and $j \geq k$ then everything is clear. Else either $a^{i}$ or $b^{j}$ is a part of the "bad" block in the beginning (or in the end) of $W$. Hence $Q$ begins (terminates) with a "bad" block of length not more then $\left|A_{k}\right|-i\left(\left|A_{k}\right|-j\right)$. Consequently, by the construction if the property is true for $W_{1}$ and $W_{2}$ then it is true for $\left(W_{1}, W_{2}\right)$. Now observe that $A_{m+1}$ is obtained by adding to $A_{m} 0^{m} 1^{m}$ combination blocks $c\left(W_{1}^{*}, W_{2}^{*}\right)$ of all pairs $\left(W_{1}^{*}, W_{2}^{*}\right)$ of subblocks of $A_{m}$ concatenated in a proper way. So, the property holds for $A_{m+1}$.

Put $(Y, F):=\left(\overline{\operatorname{orb}_{\sigma}(x)}, \sigma\right)$. Now let us check that it has the required properties. In any subblock of $x$ with length more than $\left|A_{k}\right|+2 k-1$ one can find $0^{k}$ or $1^{k}$. Fix $y \in Y$. Any starting block of $y$ can be definitely found as a block in $x$. Hence for any $k \in \mathbb{N}$ there exist big enough $M$ such that the starting block of $y$ consists either $0^{k}$ or $1^{k}$. Moreover, for each $k \in \mathbb{N}$ both $0^{k}$ and $1^{k}$ appear in $x$. Hence, both $0^{\infty}$ and $1^{\infty}$ are fixed points of $\left(\overline{\operatorname{orb}_{\sigma}(x)}, \sigma\right)$, and there is no other minimal sets in it.

Recall that the base for the open sets in $\Sigma$ is given by the collection of all cylinder sets $C\left[c_{0} c_{1} c_{2} \ldots c_{m}\right]=\left\{x \in \Sigma: x_{i}=c_{i}\right.$ for $\left.0 \leq i \leq m\right\}$. We are going to prove that for any $U, V \subset Y N_{F}(U, V)$ is cofinite. By the latter, there exist subblocks $W_{1}$, $W_{2}$ of $x$ such that $Y \cap C\left[W_{1}\right] \subset U$ and $Y \cap C\left[W_{2}\right] \subset V$. Both of $W_{1}, W_{2}$ belong to $A_{m}$ for big enough $m$. We write $W_{1}$ and $W_{2}$ in the form as above: $W_{1}=a^{i_{1}} Q_{1} b^{j_{1}}$ and $W_{2}=c^{i_{2}} Q_{2} d^{j_{2}}$ with $a, b, c, d \in\{0,1\}$ and $i_{1}, i_{2} \in \mathbb{N}, j_{1}, j_{2} \in \mathbb{Z}_{+}$, whereas $Q_{1}$ and $Q_{2}$ are possible empty subblocks. If $b=c$, then for each $l \geq m$ there exists a combination block $c_{l}\left(W_{1}, W_{2}\right)$ containing the subblock $W_{1} b^{l} W_{2}$, and hence $N_{F}\left(C\left[W_{1}\right] \cap Y, C\left[W_{2}\right] \cap Y\right) \supset\left\{m+\left|W_{1}\right|, m+\left|W_{1}\right|+1, \ldots\right\}$; if $b \neq c$, then for each $l \geq$ $m$ there exists a combination block $c_{l}\left(W_{1}, W_{2}\right)$ containing the subblocks $W_{1} b^{l} c^{l} W_{2}$ and $W_{1} b^{l} 1 c^{l} W_{2}$, and hence $N_{F}\left(\left[W_{1}\right] \cap Y,\left[W_{2}\right] \cap Y\right) \supset\left\{2 m+\left|W_{1}\right|, 2 m+\left|W_{1}\right|+1, \ldots\right\}$. This shows that the system $(Y, F)$ is topologically mixing. 
Now we shall finish the construction by the second step. Define $\left(X^{\prime}, T^{\prime}\right)=$ $\prod_{1}^{\infty}(Y, F)$. Let us check that the system $\left(X^{\prime}, T^{\prime}\right)$ is topologically mixing, for which the middle-third Cantor set $C$ is the set of all its minimal points and its each minimal point is a fixed point. Note that there exists a continuous surjection $h: C \rightarrow Z$ (see for example [26, Page 165-166, Problem O]), and then we consider the quotient system $(X, T)$ with $X=X^{\prime} / \sim$ equipped with the action induced naturally from $T^{\prime}$, where the closed positively $T^{\prime} \times T^{\prime}$-invariant equivalence relation $\sim$ is defined via $x \sim y$ if and only if $x=y \in X^{\prime} \backslash Z$ or $h(x)=h(y)$ for $x, y \in Z$. Then the system $(X, T)$ has the required properties.

The following result shows that in general there is no topological structure similar to Lemma 6.2 for the $\omega_{\mathcal{N}_{T}}$-limit sets.

Theorem 6.4. For any given compact metric space $Z$, there exists a non totally transitive, transitive compact system $(X, T)$ such that, $Z$ can be realized as the set of all its minimal points with its each minimal point being a fixed point, furthermore, $Z$ is realized as $\omega_{\mathcal{N}_{T}}(x)$ for some $x \in X$.

Proof. The idea of the proof is very similar to that of the first part (proximal case) of [23, Theorem 3.14]. We fix the given compact metric space $Z$.

By Proposition 6.3 there exists a topologically mixing system $(Y, F)$, with $Z$ realized as the set of all of its minimal points where its each minimal point is a fixed point. We take a copy of it: the system $\left(Y_{c}, F_{c}\right)$, with $Z_{c}$ realized as the set of all of its minimal points where its each minimal point is a fixed point, in particular, $Y_{c}=\left\{y_{c}: y \in Y\right\}$ with $F_{c}\left(y_{c}\right)=(F y)_{c}$ for all $y \in Y$ and $Z_{c}=\left\{z_{c}: z \in Z\right\}$. Suppose that $Y$ and $Y_{c}$ are disjoint and consider the wedge sum $X:=Y \vee Y_{c}$, i.e., the quotient space of the disjoint union of $Y$ and $Y_{c}$ by identifying $z$ and $z_{c}$ for each $z \in Z$, and then both topological spaces $Y$ and $Y_{c}$ look like subspaces of the wedge sum with the subspace topology. A self-map $T$ over $X$ is defined as follows:

$$
T: x \mapsto\left\{\begin{array}{ll}
F_{c} y_{c} & \text { if } x=y \in Y \\
F y & \text { if } x=y_{c} \in Y_{c}
\end{array}, \forall x \in X .\right.
$$

From the above construction it is not hard to show that the map $T: X \rightarrow X$ is a continuous surjection, and the system $(X, T)$ is not totally transitive by observing that $T^{2} Y \subset Y$ and $T^{2} Y_{c} \subset Y_{c}$. Then we obtain a non totally transitive system $(X, T)$ such that $Z$ is realized as the set of all of its minimal points with its each minimal point being a fixed point.

Firstly we show that $Z \supset \omega_{\mathcal{N}_{T}}(x)$ for all $x \in X$. It is easy to check $\omega_{\mathcal{N}_{T}}(x)=\{x\}$ for the case of $x \in Z$. Now assume that $x \in Y \backslash Y_{c}$. For any $z \in Y \backslash Y_{c}$, take a neighborhood $G_{z} \subset Y \backslash Y_{c}$. From the construction of $T$ one has that $N_{T}\left(x, G_{z}\right)$ consists of only even numbers. Now in $Y$ we take an opene subset $U$ of $X$ and in $Y_{c}$ we take an opene subset $V$ of $X$. Again from the construction of $T$ one has that $N_{T}(U, V)$ consists of only odd numbers. In particular, $N_{T}\left(x, G_{z}\right) \cap N_{T}(U, V)=\varnothing$, and then $z \notin \omega_{\mathcal{N}_{T}}(x)$. We can prove similarly that $\omega_{\mathcal{N}_{T}}(x) \cap Y_{c} \backslash Y=\varnothing$. Summing up, we obtain $\omega_{\mathcal{N}_{T}}(x) \subset Z$. The case of $x \in Y_{c} \backslash Y$ can be done similarly.

Now we prove the following claim:

Claim. For each $x \in X$, if $x=y \in Y$ and $p \in Z \cap \omega_{\mathcal{N}_{F 2}}(y)$ then, as a point in $X$, $p$ belongs to $\omega_{\mathcal{N}_{T}}(x)$. 
Proof of Claim. Let $U_{p}$ be an open subset of $X$ containing $p$, and clearly $U_{p}$ may be also viewed as an open subset of $Y$ containing $p$. Now for any given opene subsets $U$ and $V$ of $X$ : if both $U$ and $V$ can be viewed as opene subsets of $Y$, then we can take $n \in N_{F^{2}}\left(y, U_{p}\right) \cap N_{F^{2}}(U, V)$ by the assumption $p \in \omega_{\mathcal{N}_{F^{2}}}(y)$ and hence $2 n \in N_{T}\left(y, U_{p}\right) \cap N_{T}(U, V)$; if both $U$ and $V$ can be viewed as opene subsets of $Y_{c}$, then both $T^{-1} U$ and $T^{-1} V$ can be viewed as opene subsets of $Y$ and hence $N_{T}\left(y, U_{p}\right) \cap N_{T}(U, V)=N_{T}\left(y, U_{p}\right) \cap N_{T}\left(T^{-1} U, T^{-1} V\right) \neq \varnothing$; if $U$ and $V$ can be viewed as opene subsets of $Y$ and $Y_{c}$, respectively, noting $p \in Z$ and hence $T p=p$, there is an opene subset $V_{p}$ of $X$ containing $p$ such that $T V_{p} \subset U_{p}$, and then by the above reasoning we may take $n \in N_{T}\left(y, V_{p}\right) \cap N_{T}\left(U, T^{-1} V\right)$, and hence $n+1 \in N_{T}\left(y, U_{p}\right) \cap N_{T}(U, V)$; it can be treated similarly the other case that $U$ and $V$ can be viewed as opene subsets of $Y_{c}$ and $Y$, respectively.

We continue our proof. As $(Y, F)$ is topologically mixing, the system $\left(Y, F^{2}\right)$ is weakly mixing, and then by [23, Proposition 3.8] we may choose $x^{*} \in Y$ such that $\omega_{\mathcal{N}_{F^{2}}}\left(x^{*}\right)=Y$, in particular, $\omega_{\mathcal{N}_{F^{2}}}\left(x^{*}\right) \supset Z$. Thus, by the above Claim, we obtain $Z \subset \omega_{\mathcal{N}_{T}}\left(x^{*}\right)$ and hence $Z=\omega_{\mathcal{N}_{T}}\left(x^{*}\right)$.

Finally we prove that the system $(X, T)$ is transitive compact, which finishes the proof. Let $x \in X$. If $x \in Z$ then $\omega_{\mathcal{N}_{T}}(x) \neq \emptyset$ as $\omega_{\mathcal{N}_{T}}(x)=\{x\}$. If $x \in Y \backslash Y_{c}$, applying [23, Proposition 3.5] to the weakly mixing system $\left(Y, F^{2}\right)$ one has that $\omega_{\mathcal{N}_{F^{2}}}(x)$ is a nonempty $F^{2}$-invariant subset of $Y$, and then $\omega_{\mathcal{N}_{F^{2}}}(x) \cap Z \neq \varnothing$ as $Z$ is the set of all of minimal points in $(Y, F)$, hence $\omega_{\mathcal{N}_{T}}(x) \neq \emptyset$ follows from the above Claim. The case of $x \in Y_{c} \backslash Y$ can be done similarly.

Note that a dynamical system is proximal if and only if it contains the unique fixed point, which is the only minimal point of the system [4]. Thus, as a direct corollary of Lemma 6.2 and Theorem 6.4, we have:

Corollary 6.5. There exists a non-proximal, non totally transitive, transitive compact system $(X, T)$ and a point $x_{0} \in X$ such that $\omega_{\mathcal{N}_{T}}\left(x_{0}\right) \neq \omega_{T}(x)$ for all $x \in X$.

Nevertheless is still open the following

Question B. Let $(X, T)$ be a weakly mixing system. Is there a point $x \in X$ and $2 \leq \alpha \leq \aleph_{0}$ such that $\omega_{\mathcal{N}_{T}}(x)$ can be decomposed into $\alpha$ disjoint closed, nonempty, positively T-invariant subsets?

At the end of this section let us prove one more chaotical property of transitive compact systems in additional to already known in [23].

Recall that a pair of points $x, y \in X$ is asymptotic if $\lim _{n \rightarrow \infty} d\left(T^{n} x, T^{n} y\right)=0$. Denote by $\operatorname{Asym}_{T}(X)$ the set of all asymptotic pairs of points. Any pair $(x, y) \in$ $\operatorname{Prox}_{T}(X) \backslash \operatorname{Asym}_{T}(X)$ is called a Li-Yorke pair. Recall that a dynamical system $(X, T)$ is Li-Yorke chaotic if there exists an uncountable set $S \subset X$ with $(S \times S) \backslash$ $\Delta_{2}(X) \subset \operatorname{Prox}_{T}(X) \backslash \operatorname{Asym}_{T}(X)$, where $\Delta_{2}(X)=\{(x, x): x \in X\}$.

Proposition 6.6. Each transitive compact system $(X, T)$ is Li-Yorke chaotic.

Proof. Clearly $(X, T)$ is transitive. Observe that we have assumed the state space to be not a singleton and in fact a compact metric space without isolated points, then $(X, T)$ is a transitive system with $X$ infinite. Thus, the subset $\operatorname{Asym}_{T}(X)$ is a fist category subset of $X \times X$ by [25, Corollary 2.2]. It is easy to show that $\operatorname{Prox}_{T}(X)$ is a $G_{\delta}$ subset of $X \times X$, and applying [23, Proposition 3.7] to the transitive compact system $(X, T)$ we have that $\operatorname{Prox}_{T}(x)$ is a dense subset of $X$ for each $x \in X$. Thus 
$\operatorname{Prox}_{T}(X)$ is a dense $G_{\delta}$ subset of $X \times X$, and then $\operatorname{Prox}_{T}(X) \backslash \operatorname{Asym}_{T}(X)$ is a second category subset of $X \times X$. Now applying the well-known Mycielski Theorem [32, Theorem 1] we obtain an uncountable subset $S \subset X$ with $(S \times S) \backslash \Delta_{2}(X) \subset$ $\operatorname{Prox}_{T}(X) \backslash \operatorname{Asym}_{T}(X)$. That is, $(X, T)$ is Li-Yorke chaotic.

\section{WEAK TRANSITIVE COMPACTNESS AND SENSITIVITY FOR LINEAR OPERATORS}

In this section we are considering the dynamics of linear operators on infinite dimensional spaces in relation to the properties studied in previous sections. More precisely, we will show the equivalence of the topological weak mixing property with a weak version of transitive compactness. We obtain some results on transitive sensitivity too.

One should keep in mind that, for a linear dynamical system $(X, T)$, where $X$ is an infinite dimensional space, neither compactness nor even local compactness of $X$ is satisfied. In particular, we are interested in the case where $X$ is an infinite dimensional separable Banach space and $T: X \rightarrow X$ is a continuous linear map (in short, operator). In this framework, we will just write $(X, T)$ is an infinite dimensional linear dynamical system. We recall that $X$ is a Banach space if it is a vector space endowed with a norm $\|\cdot\|$ such that $X$ with the associated distance $d(x, y):=\|x-y\|$ becomes a complete metric space. It is well known that $T: X \rightarrow$ $X$ is an operator if and only if $\|T\|:=\sup \{\|T x\|:\|x\| \leq 1\}<\infty$. We refer the reader to the books [7] and [19] for the theory of linear dynamics.

Note that all notations and concepts discussed in previous sections can be introduced into linear dynamics. We also introduce a weak version of dynamical compactness. A linear system $(X, T)$ is called weakly dynamically compact with respect to the family $\mathcal{F}$ if there exists a dense subset $X_{0} \subset X$ such that the $\omega_{\mathcal{F}-}$ limit set $\omega_{\mathcal{F}}(x)$ is nonempty for all $x \in X_{0}$. In particular, $(X, T)$ is called weakly transitive compact, if there exists a dense subset $X_{0} \subset X$ such that for any point $x \in X_{0}$ the $\omega_{\mathcal{N}_{T}}$-limit set $\omega_{\mathcal{N}_{T}}(x)$ is nonempty, in other words, for any point $x \in X_{0}$ there exists a point $z \in X$ such that

$$
N_{T}(x, G) \cap N_{T}(U, V) \neq \varnothing
$$

for any neighborhood $G$ of $z$ and any opene subsets $U, V$ of $X$.

Theorem 7.1. Let $(X, T)$ be an infinite dimensional linear system. Then $(X, T)$ is weakly mixing if and only if it is weakly transitive compact.

Proof. Sufficiency. Suppose that $(X, T)$ is weakly transitive compact. Let $X_{0} \subset X$ be a dense subset such that, for each $x \in X_{0}$, there exists $z(x) \in X$ such that

$$
N_{T}(x, G) \cap N_{T}(U, V) \neq \varnothing
$$

for any neighborhood $G$ of $z(x)$ and opene $U, V \subset X$. As $(X, T)$ is obviously transitive, by [18, Theorem 5] (see also [19, Theorem 2.45]) to obtain the weak mixing property we just need to show that, for each opene $U \subset X$ and 0-neighbourhood $W$, there is a continuous map $S: X \rightarrow X$ commuting with $T$ such that

$$
S(U) \cap W \neq \varnothing \quad \text { and } \quad S(W) \cap U \neq \varnothing .
$$

Given an opene subset $U$ of $X$ and a 0 -neighborhood $W$, we fix $x \in U \cap X_{0}$ and $z(x) \in X$ accordingly to the weak transitive compactness of $(X, T)$. Since 
0 -neighbourhoods are absorbing, we find a scalar $\lambda \neq 0$ such that $\lambda z(x) \in W$. Let $G$ be a neighbourhood of $z(x)$ such that $\lambda G \subset W$. By the hypothesis we can find

$$
m \in N_{T}(x, G) \cap N_{T}(\lambda W, U) .
$$

That is, $T^{m} x \in G$ and so $\lambda T^{m} x \in W$; additionally, there exists $w \in W$ with $T^{m} \lambda w \in U$. Now pick $S:=\lambda T^{m}$, we have that $S$ commutes with $T$ and the property (7.1) is satisfied, therefore the system is weakly mixing.

Necessity. Conversely, under the assumption of the weak mixing property for $(X, T)$, we know by [8, Theorem 2.3] (see also [19, Theorem 3.15]) that there exists an increasing sequence $\left\{n_{k}: k \in \mathbb{N}\right\} \subset \mathbb{N}$ and a dense subset $X_{0} \subset X$ such that $T^{n_{k}} x \rightarrow 0$ for each $x \in X_{0}$ and, for arbitrary opene $U, V \subset X$, we can find $k \in \mathbb{N}$ such that $T^{n_{k}}(U) \cap V \neq \varnothing$. Thus, we obtain easily that $(X, T)$ is weakly transitive compact by selecting $z(x)=0$ for every $x \in X_{0}$.

Concerning sensitivity, the situation is more complicated and, although we obtain some advances, three related problems are left open.

Proposition 7.2. Let $(X, T)$ be an infinite dimensional linear, topologically transitive system. Then $(X, T)$ is thickly multi-sensitive, that is, there exists $\delta>0$ such that $\bigcap_{i=1}^{k} S_{T}\left(U_{i}, \delta\right)$ is thick for any finite collection of opene $U_{1}, \ldots, U_{k} \subset X$.

Proof. Let $U_{1}, \ldots, U_{k}$ be opene sets, and let $m \in \mathbb{N}$. Pick points $x_{1}, \ldots, x_{k}$ such that $x_{i} \in U_{i}$ and choose $\varepsilon>0$ such that $B_{\varepsilon}\left(x_{i}\right) \subset U_{i}$, where $B_{\varepsilon}\left(x_{i}\right)$ is the open ball of radius $\varepsilon$ centered at $x_{i}$, for all $i \in\{1, \ldots, k\}$. By a hypercyclic vector we mean that its orbit is dense in the space $X$. Take a hypercyclic vector $u \in B_{\varepsilon}(0)$ by [19, Theorem 2.19], and let $y_{i}=x_{i}+u$. Then $y_{i} \in U_{i}$ by the construction. Since $u$ is hypercyclic there is $n \in \mathbb{N}, n>m$, such that $\left\|T^{n} u\right\|>(\|T\|+1)^{m}$. Then $\rho\left(T^{n-j} x_{i}, T^{n-j} y_{i}\right)=\left\|T^{n-j}\left(x_{i}-y_{i}\right)\right\|=\left\|T^{n-j} u\right\|>(\|T\|+1)^{m-j}>1$ for all $i=1, \ldots, k$ and $j=0, \ldots, m-1$. Hence $\{n, n-1, \ldots, n-m+1\} \subset \bigcap_{i=1}^{k} S_{T}\left(U_{i}, 1\right)$, and therefore $(X, T)$ is thickly multi-sensitive.

Proposition 7.3. Let $(X, T)$ be an infinite dimensional linear system. Then the following conditions are equivalent:

(1) For each $\delta>0,(X, T)$ is transitively sensitive with a sensitive constant $\delta$.

(2) There exists $\delta_{0}>0$ such that $(X, T)$ is transitively sensitive with a sensitive constant $\delta_{0}$.

(3) There exists $\delta_{0}>0$ such that $S_{T}\left(W_{0}, \delta_{0}\right) \cap N_{T}(U, V) \neq \varnothing$ for any opene subsets $U, V$ of $X$ and any 0-neighbourhood $W_{0}$.

Proof. We just need to show $(3) \Rightarrow(1)$. Indeed, let $\delta>0$ be arbitrary, and fix arbitrary opene $U, V, W$ of $X$. We select $\varepsilon>0$ and $x \in W$ such that $x+B_{\varepsilon}(0) \subset W$. Observing $S_{T}\left(\lambda W_{0}, \lambda \delta_{0}\right)$ for any scalar $\lambda \neq 0$, and so without loss of generality we assume $\delta>\delta_{0}$. Let $0<\varepsilon^{\prime}<\frac{\delta_{0} \varepsilon}{\delta}$, and set $W_{0}=B_{\varepsilon^{\prime}}(0)$. By the hypothesis there are $y, z \in W_{0}$ and $n \in N_{T}(U, V)$ such that $\left\|T^{n} y-T^{n} z\right\|>\delta_{0}$. Set $y^{\prime}=x+\frac{\delta}{\delta_{0}} y$ and $z^{\prime}=x+\frac{\delta}{\delta_{0}} z$. We have $y^{\prime}, z^{\prime} \in W$ and $\left\|T^{n} y^{\prime}-T^{n} z^{\prime}\right\|>\delta$. As opene $U, V, W \subset X$ are arbitrary, $(X, T)$ is transitively sensitive with a sensitive constant $\delta$.

In this framework the weak mixing property implies transitive sensitivity too. The following result establishes a very close connection of transitivity with transitive sensitivity. We do not know, however, whether every transitive linear system is transitively sensitive. 
Proposition 7.4. Let $(X, T)$ be an infinite dimensional linear, topologically transitive system. If $(X, T)$ is not transitively sensitive, then there exists a dense open subset $U_{0} \subset X$ such that every $x \in U_{0}$ has a dense orbit.

Proof. If $(X, T)$ is not transitively sensitive, by Proposition 7.3 we find opene $U, V$ of $X$ and $\delta>1$ such that $\left\|T^{n} x\right\| \leq \delta$ whenever $n \in N_{T}(U, V)$ and $\|x\| \leq 1$. We fix an arbitrary opene $V^{\prime} \subset V$ and select an opene $\widehat{V} \subset V^{\prime}$ and $\varepsilon>0$ such that $\widehat{V}+B_{\varepsilon}(0) \subset V^{\prime}$. Given $u \in U$, there is $\varepsilon^{\prime}<\frac{\varepsilon}{\delta}$ such that $U^{\prime}:=u+B_{\varepsilon^{\prime}}(0) \subset U$. Since $T$ is transitive, there exists $m \in N_{T}\left(U^{\prime}, \widehat{V}\right) \subset N_{T}(U, V)$. That is, we find $u^{\prime}=u+w \in U^{\prime}$ with $\|w\|<\varepsilon^{\prime}$ and $T^{m} u^{\prime} \in \widehat{V}$. By the assumption $\left\|T^{m} w\right\| \leq \delta \varepsilon^{\prime}<$ $\varepsilon$. Therefore, $T^{m} u=T^{m} u^{\prime}-T^{m} w \in \widehat{V}+B_{\varepsilon}(0) \subset V^{\prime}$. Since $u \in U$ and opene $V^{\prime} \subset V$ are arbitrary, we obtain that the orbit of every element in $U$ is somewhere dense, thus everywhere dense by transitivity of the system. Finally, the open set $U_{0}:=\bigcup_{n \in \mathbb{N}} T^{-n}(U)$ is dense, and every element in $U_{0}$ has a dense orbit.

There are (very difficult) examples of linear systems $(X, T)$ such that every nonzero element has a dense orbit [36], but it seems to unknown whether every linear system that admits an open set of elements whose orbit is dense is so that every non-zero element has a dense orbit. It is also worthy to mention that there are (also rare) examples of transitive but not weakly mixing linear systems [10] (see also [7]), but as far as we know there are no examples of transitive non-weakly mixing linear systems such that every non-zero element has a dense orbit.

Concerning weak disjointness, observe that for each separable Banach space the family of all opene subsets admits a countable base, and then it is a routine to show that Theorem 5.2 holds true within linear systems too. Note that the intersection of finitely many thickly syndetically sets is still thickly syndetic, and that an interesting property is that every topologically ergodic linear system $(X, T)$ (i.e., each element of $\mathcal{N}_{T}$ is a syndetic set) satisfies that each element of $\mathcal{N}_{T}$ is actually a thickly syndetic set (see the exercises in [19, Chapter 2]). Thus any finite family $\left(X_{1}, T_{1}\right), \ldots,\left(X_{k}, T_{k}\right)$ of topologically ergodic linear systems is weakly disjoint and, moreover, the product system $\left(X_{1} \times \cdots \times X_{k}, T_{1} \times \cdots \times T_{k}\right)$ is topologically ergodic.

\section{REFERENCES}

1. Ethan Akin, Recurrence in topological dynamics, The University Series in Mathematics, Plenum Press, New York, 1997, Furstenberg families and Ellis actions. MR 1467479 (2000c:37014)

2. Ethan Akin, Joseph Auslander, and Kenneth Berg, When is a transitive map chaotic?, Convergence in ergodic theory and probability (Columbus, OH, 1993), Ohio State Univ. Math. Res. Inst. Publ., vol. 5, de Gruyter, Berlin, 1996, pp. 25-40. MR 1412595 (97i:58106)

3. Ethan Akin and Eli Glasner, Residual properties and almost equicontinuity, J. Anal. Math. 84 (2001), 243-286. MR 1849204 (2002f:37020)

4. Ethan Akin and Sergiı̌ Kolyada, Li-Yorke sensitivity, Nonlinearity 16 (2003), no. 4, 14211433. MR 1986303 (2004c:37016)

5. Joseph Auslander, Minimal flows and their extensions, North-Holland Mathematics Studies, vol. 153, North-Holland Publishing Co., Amsterdam, 1988, Notas de Matemática [Mathematical Notes], 122. MR 956049 (89m:54050)

6. Joseph Auslander and James A. Yorke, Interval maps, factors of maps, and chaos, Tôhoku Math. J. (2) 32 (1980), no. 2, 177-188. MR 580273 (82b:58049)

7. Frédéric Bayart and Étienne Matheron, Dynamics of linear operators, Cambridge Tracts in Mathematics, vol. 179, Cambridge University Press, Cambridge, 2009. MR 2533318 
8. Juan Bès and Alfredo Peris, Hereditarily hypercyclic operators, J. Funct. Anal. 167 (1999), no. 1, 94-112. MR 1710637

9. François Blanchard and Wen Huang, Entropy sets, weakly mixing sets and entropy capacity, Discrete Contin. Dyn. Syst. 20 (2008), no. 2, 275-311. MR 2358261

10. Manuel de la Rosa and Charles Read, A hypercyclic operator whose direct sum $T \oplus T$ is not hypercyclic, J. Operator Theory 61 (2009), no. 2, 369-380. MR 2501011

11. Yael Naim Dowker and F. G. Friedlander, On limit sets in dynamical systems, Proc. London Math. Soc. (3) 4 (1954), 168-176. MR 0061820 (15,889e)

12. Tomasz Downarowicz, Survey of odometers and Toeplitz flows, Algebraic and topological dynamics, Contemp. Math., vol. 385, Amer. Math. Soc., Providence, RI, 2005, pp. 7-37. MR 2180227 (2006f:37009)

13. R. E. Edwards, Functional analysis, Dover Publications, Inc., New York, 1995, Theory and applications, Corrected reprint of the 1965 original. MR 1320261

14. Harry Furstenberg, Disjointness in ergodic theory, minimal sets, and a problem in Diophantine approximation, Math. Systems Theory 1 (1967), 1-49. MR 0213508 (35 \#4369)

15. _ Recurrence in ergodic theory and combinatorial number theory, Princeton University Press, Princeton, N.J., 1981, M. B. Porter Lectures. MR 603625 (82j:28010)

16. Harry Furstenberg and Benjamin Weiss, Topological dynamics and combinatorial number theory, J. Analyse Math. 34 (1978), 61-85 (1979). MR 531271 (80g:05009)

17. Eli Glasner and Benjamin Weiss, Sensitive dependence on initial conditions, Nonlinearity 6 (1993), no. 6, 1067-1075. MR 1251259 (94j:58109)

18. Karl-G. Grosse-Erdmann and Alfredo Peris, Weakly mixing operators on topological vector spaces, Rev. R. Acad. Cienc. Exactas Fís. Nat. Ser. A Math. RACSAM 104 (2010), no. 2, 413-426. MR 2757249

19. Karl-G. Grosse-Erdmann and Alfredo Peris Manguillot, Linear chaos, Universitext, Springer, London, 2011. MR 2919812

20. John Guckenheimer, Sensitive dependence to initial conditions for one-dimensional maps, Comm. Math. Phys. 70 (1979), no. 2, 133-160. MR 553966 (82c:58037)

21. James D. Halpern, Bases in vector spaces and the axiom of choice, Proc. Amer. Math. Soc. 17 (1966), 670-673. MR 0194340

22. Wei Hong He and Zuo Ling Zhou, A topologically mixing system whose measure center is a singleton, Acta Math. Sinica (Chin. Ser.) 45 (2002), no. 5, 929-934. MR 1941883 (2003j:37017)

23. Wen Huang, Danylo Khilko, Sergiŭ Kolyada, and Guohua Zhang, Dynamical compactness and sensitivity, J. Differential Equations 260 (2016), no. 9, 6800-6827. MR 3461085

24. Wen Huang, Sergiı̌ Kolyada, and Guohua Zhang, Analogues of Auslander-Yorke theorems for multi-sensitivity, Ergodic Theory Dynam. Systems, published online on 22 September 2016, pp. 115. doi: 10.1017/etds.2016.48.

25. Wen Huang and Xiangdong Ye, Devaney's chaos or 2-scattering implies Li-Yorke's chaos, Topology Appl. 117 (2002), no. 3, 259-272. MR 1874089 (2003b:37017)

26. John L. Kelley, General topology, Springer-Verlag, New York-Berlin, 1975, Reprint of the 1955 edition [Van Nostrand, Toronto, Ont.], Graduate Texts in Mathematics, No. 27. MR 0370454 (51 \#6681)

27. Sergiĭ Kolyada, L’ubomír Snoha, and Serger Trofimchuk, Noninvertible minimal maps, Fund. Math. 168 (2001), no. 2, 141-163. MR 1852739 (2002j:37017)

28. Jian Li, Transitive points via Furstenberg family, Topology Appl. 158 (2011), no. 16, 22212231. MR 2831911 (2012m:37012)

29. Jian Li and Xiang Dong Ye, Recent development of chaos theory in topological dynamics, Acta Math. Sin. (Engl. Ser.) 32 (2016), no. 1, 83-114. MR 3431162

30. Heng Liu, Li Liao, and Lidong Wang, Thickly Syndetical Sensitivity of Topological Dynamical System, Discrete Dyn. Nat. Soc. (2014), Art. ID 583431, 4. MR 3200824

31. T. K. Subrahmonian Moothathu, Stronger forms of sensitivity for dynamical systems, Nonlinearity 20 (2007), no. 9, 2115-2126. MR 2351026 (2008j:37014)

32. Jan Mycielski, Independent sets in topological algebras, Fund. Math. 55 (1964), 139-147. MR 0173645

33. Piotr Oprocha and Guohua Zhang, On local aspects of topological weak mixing in dimension one and beyond, Studia Math. 202 (2011), no. 3, 261-288. MR 2771654

34. - On local aspects of topological weak mixing, sequence entropy and chaos, Ergodic Theory Dynam. Systems 34 (2014), no. 5, 1615-1639. MR 3255435 
35. K. E. Petersen, Disjointness and weak mixing of minimal sets, Proc. Amer. Math. Soc. 24 (1970), 278-280. MR 0250283 (40 \#3522)

36. C. J. Read, The invariant subspace problem for a class of Banach spaces. II. Hypercyclic operators, Israel J. Math. 63 (1988), no. 1, 1-40. MR 959046

37. David Ruelle, Dynamical systems with turbulent behavior, Mathematical problems in theoretical physics (Proc. Internat. Conf., Univ. Rome, Rome, 1977), Lecture Notes in Phys., vol. 80, Springer, Berlin-New York, 1978, pp. 341-360. MR 518445 (81e:58032)

38. A. N. Šarkovskiı̌, Continuous mapping on the limit points of an iteration sequence, Ukrain. Mat. Ž. 18 (1966), no. 5, 127-130. MR 0206916 (34 \#6732)

39. Benjamin Weiss, A survey of generic dynamics, Descriptive set theory and dynamical systems (Marseille-Luminy, 1996), London Math. Soc. Lecture Note Ser., vol. 277, Cambridge Univ. Press, Cambridge, 2000, pp. 273-291. MR 1774430

School of Mathematical Sciences, University of Science and Technology of China, Hefei, Anhui 230026, China

Department of Mathematics, Sichuan University, Chengdu, Sichuan 610064, China

E-mail address: wenh@mail.ustc.edu.cn

DÉPARTEMENT De mathématiques et applications, École noRmale SupŔieure, 45 RUE D'Ulm, 75005 Paris, France

E-mail address: danylo.khilko@ens.fr

Institute of Mathematics, NASU, Tereshchenkivs'Ka 3, 01601 Kyiv, Ukraine

E-mail address: skolyada@imath.kiev.ua

Institut Universitari de Matemàtica Pura i Aplicada, Universitat Politècnica de València, Edifici 8E, Cub F, 4a planta, 46022 València, Spain

E-mail address: aperis@mat.upv.es

School of Mathematical Sciences and LMns, Fudan University and Shanghai Center for Mathematical Sciences, Shanghai 200433, China

E-mail address: chiaths.zhang@gmail.com 